\title{
THE GENUS OF A MAP
}

BY

SARA HURVITZ

\begin{abstract}
The elements $\left[f^{\prime}\right]\left(f^{\prime}: X^{\prime} \rightarrow Y^{\prime}\right)$ of the genus $-G(f)$ of a map $f: X \rightarrow Y$ are equivalence classes of homotopy classes of maps $f^{\prime}$ which satisfy: For every prime $p$ there exist homotopy equivalences $h_{p}: X_{p}^{\prime} \rightarrow X_{p}$ and $k_{p}: Y_{p}^{\prime} \rightarrow Y_{p}$ so that $f_{p} h_{p} \sim k_{p} f_{p}^{\prime}$. The genus of $f$ under $X-G^{X}(f)$ and the genus of $f$ over $Y-G_{Y}(f)$ are defined similarly.

In this paper we prove that under certain conditions on $f$, the sets $G(f), G^{X}(f)$ and $G_{Y}(f)$ are finite and admit an abelian group structure. We also compare the genus of $f$ to those of $X$ and $Y$, calculate it for some principal fibrations of the form $K(G, n-1) \rightarrow X \rightarrow Y$, and deal with the noncancellation phenomenon.
\end{abstract}

1. Introduction. In this paper we use the structure of the genus of an $\boldsymbol{H}_{0}$-space, which was investigated by Zabrodsky [8], to study the structure of the genus of a map $f: X \rightarrow Y$. In some cases we calculate the genus and compare it with those of $X$ and $Y$.

All spaces considered are pointed and of the homotopy type of simply connected CW-complexes of finite type and either with a finite number of nonzero homology groups or with a finite number of nonzero homotopy groups.

Throughout this paper we work in the homotopy category. We recall that for a CW-complex $X$, the genus of $X$ is the set $G(X)$ of homotopy types of spaces $Y$ with $Y_{p} \approx X_{p}$ for every prime $p$ (where ( $)_{p}$ denotes the $p$-localization operation). We define analogously the genus $G(f)$ of $f, G^{X}(f)$-the genus of maps under $X$ and $G_{Y}(f)$ the genus of maps over $Y$.

1.1. Definition. Let $f: X \rightarrow Y$ be a map. The elements $\left[f^{\prime}\right] \in G(f)\left(f^{\prime}: X^{\prime} \rightarrow Y^{\prime}\right)$ are equivalence classes of homotopy classes of $f^{\prime}$ which satisfy: For every prime $p$ there exist homotopy equivalences $h_{p}: X_{p}^{\prime} \rightarrow X_{p}$ and $k_{p}: Y_{p}^{\prime} \rightarrow Y_{p}$ so that $f_{p} h_{p} \sim k_{p} f_{p}^{\prime}$. (We denote the genus of $f$ either by $G(f)$ or by $G(X, Y, f)$.)

The elements $\left[f^{\prime}\right] \in G^{X}(f)\left(f^{\prime}: X \rightarrow Y^{\prime}\right)$ are equivalence classes of homotopy classes of maps $f^{\prime}$ which satisfy: For every prime $p$ there exists a homotopy equivalence $k_{p}: Y_{p}^{\prime} \rightarrow Y_{p}$ so that $k_{p} f_{p}^{\prime} \sim f_{p}$. (Two maps $f_{i}: X \rightarrow Y_{i}, i=1,2$, are equivalent under $X$ if there exists a homotopy equivalence $k: Y_{1} \rightarrow Y_{2}$ with $k f_{1} \sim f_{2}$.)

The elements $\left[f^{\prime}\right] \in G_{Y}(f)\left(f^{\prime}: X^{\prime} \rightarrow Y\right)$ are equivalence classes of homotopy classes of maps $f^{\prime}$ so that for every prime $p$ there exists a homotopy equivalence $h_{p}$ : $X_{p}^{\prime} \rightarrow X_{p}$ so that $f_{p} h_{p} \sim f_{p}^{\prime}$.

To state the main results of this study we need the following notations: Let $t$ be an integer, $X$ a space and $f: X \rightarrow Y$ a map. Denote by $Z_{t}$ the group $Z / t Z$, by $Z_{t}^{*}$

Received by the editors December 5, 1978 and, in revised form, September 26, 1979.

AMS (MOS) subject classifications (1970). Primary 55D99. 
the units in $Z_{t}$, by $l(X)$ the number of integers $n$ with $Q H^{n}(X, Q) \neq 0$ and by $[f, f]_{t}$ the set of pairs $(h, k)$ of $t$-equivalences $h: X \rightarrow X, k: Y \rightarrow Y$ satisfying $k f \sim f h$. (A $t$-equivalence of $X$ is a map $f: X \rightarrow X$ so that $H^{*}(f, Z) \otimes Z_{p}$ is an isomorphism for every prime $p$ which divides $t$.)

THEOREM I. Let $f: X \rightarrow Y$ be a map which satisfies one of the following conditions:

(a) $f: X \rightarrow Y$ is an $H$-map, $H^{*}(X, Q)$ and $H^{*}(Y, Q)$ are primitively generated and $H^{*}(f, Q)$ is either a monomorphism, an epimorphism, an isomorphism or zero.

(b) $X=S^{2 n-1}, Y$ is an $H$-space and $H^{*}(Y, Q)$ is primitively generated.

(c) $X=S^{2 n-1}, Y=S^{2 m-1}$.

Then $G(f)$ admits an abelian group structure and there exist integers $k$ and $\hat{t}$ (depending on $X, Y$ and $f$ ) and an exact sequence

$$
[f, f]_{\hat{i}} \stackrel{\alpha^{\prime}}{\rightarrow}\left[\left(Z_{\hat{i}}^{*}\right) / \pm 1\right]^{k} \stackrel{\hat{\xi}}{\rightarrow} G(f) \rightarrow 0
$$

where $\alpha^{\prime}$ is the composition

$$
\begin{aligned}
{[f, f]_{i} } & \rightarrow \operatorname{aut}\left(Q H^{*}(X, Z) / \text { torsion } \otimes Z_{i}\right) \times \operatorname{aut}\left(Q H^{*}(Y, Z) / \text { torsion } \otimes Z_{i}\right) \\
|\operatorname{det}| \times|\operatorname{det}| & {\left[\left(Z_{i}^{*}\right) / \pm 1\right]^{l(X)+l(Y)} \rightarrow\left[\left(Z_{i}^{*}\right) / \pm 1\right]^{k} . }
\end{aligned}
$$

THEOREM II. If $f: X \rightarrow Y$ is a map satisfying the conditions of Theorem I then $G_{Y}(f)$ admits an abelian group structure and there exist an integer $\hat{t}$ depending on $X$, $Y$ and $f$ and an exact sequence

$$
[f, f]_{\hat{i}}^{\prime} \stackrel{\alpha^{\prime}}{\rightarrow}\left[\left(Z_{\hat{t}}^{*}\right) / \pm 1\right]^{l(X)} \stackrel{\hat{\xi}}{\rightarrow} G_{Y}(f) \rightarrow 0
$$

where $[f, f]_{\hat{t}}^{\prime}=\left\{(h, 1) \in[f, f]_{\hat{t}}\right\}$.

THEOREM III. Let $f: X \rightarrow Y$ be a map satisfying one of the following conditions:

(a) $X, Y$ are $H$-spaces; $H^{*}(X, Q)$ and $H^{*}(Y, Q)$ are primitively generated, and $H^{*}(f, Q)$ is either a monomorphism, an epimorphism, an isomorphism or zero.

(b) $X=S^{2 n-1}, Y$ is an $H$-space, and $H^{*}(Y, Q)$ is primitively generated.

Then $G^{X}(f)$ admits an abelian group structure and there exist an integer $\hat{t}$ depending on $X, Y$ and $f$ and an exact sequence

$$
[f, f]_{i}^{\prime \prime} \stackrel{\alpha^{\prime}}{\rightarrow}\left[\left(Z_{i}^{*}\right) / \pm 1\right]^{l(Y)} \stackrel{\xi}{\rightarrow} G^{X}(f) \rightarrow 0
$$

where $[f, f]_{t}^{\prime \prime}=\left\{(1, k) \in[f, f]_{\hat{t}}\right\}$.

The proof of Theorem I relies heavily on the fact that for maps which satisfy the conditions of Theorem I, a map $f^{\prime}: X^{\prime} \rightarrow Y^{\prime}$ belongs to the genus of the map $f$ : $X \rightarrow Y$ iff for every prime $p$ there exist $p$-equivalences $h: X^{\prime} \rightarrow X, k: Y^{\prime} \rightarrow Y$ so that $f h \sim k f^{\prime}$. The proofs of Theorems II and III rely on similar facts. These facts are proved in $\$ 2$. The main theorems are proved in \$3. In \$4 some simple conclusions are derived. $\$ 5$ deals with the kernel of the obvious map $G(X, Y, f) \rightarrow$ $G(X) \times G(Y)$ and $\$ 6$ applies this map and the main theorems to calculate the genus of some principal fibrations of the form $K(G, n-1) \rightarrow X \rightarrow Y$. The last section, $\$ 7$, deals with the noncancellation phenomenon. 
This paper constitutes a part of the author's Ph.D. thesis written at the Hebrew University under the supervision of Professor A. Zabrodsky. It is a pleasure to thank Professor Zabrodsky for his useful advice.

2. Localization and $p$-equivalences. Let $(X, \mu)$ be an $H$-space and let $(Y, \psi)$ be a co- $H$-space. We shall denote by + the operation on $[Z, X]$ and $[Y, Z]$ induced by $\mu$ and $\psi$, respectively, by $\phi_{n}$ the $n$-power map

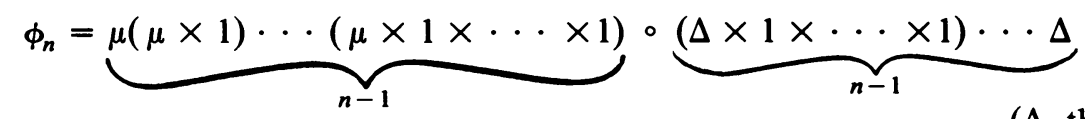

$(\Delta$-the diagonal)

and by $\eta_{n}$ the map

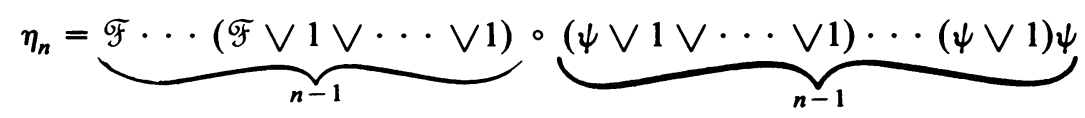

(F-the folding map).

2.1. Theorems (Hilton, Mislin, Roitberg, SEe [3, II,6]). Let $X$ be a connected $H$-space and $W$ a space with a finite number of homology groups. For every map $f$ : $W_{p} \rightarrow X_{p}$ there exist an integer $n,(n, p)=1$, and a function $\mathrm{g}: W \rightarrow X$ so that $g_{p} \sim \phi_{n} f$.

Moreover, given two functions $f, g: W \rightarrow X$ so that $f_{p} \sim g_{p}$, there exists an integer $m,(m, p)=1$, so that $\phi_{m} f \sim \phi_{m} g$.

2.2. TheOREM. Let $f: X \rightarrow Y$ be $a$ map and let $l$ be an integer. Suppose $l=$ $p_{1}^{w_{1}} \ldots p_{s}^{w_{s}}$.

(a) If $f$ is an $H$-map then:

(1) Given two spaces $X^{\prime}, Y^{\prime}$, a function $f^{\prime}: X^{\prime} \rightarrow Y^{\prime}$ and homotopy equivalences $h_{p_{i}}: X_{p_{i}}^{\prime} \rightarrow X_{p_{i}}, k_{p_{i}}: Y_{p_{i}}^{\prime} \rightarrow Y_{p_{i}}$ satisfying $f_{p_{i}} h_{p_{i}} \sim k_{p_{i}} f_{p_{i}}^{\prime}(r=1, \ldots, s)$, there exist $l-$ equivalences $h: X^{\prime} \rightarrow X, k: Y^{\prime} \rightarrow Y$ so that fh $\sim k f^{\prime}$.

(2) Given a map $f^{\prime}: X^{\prime} \rightarrow Y$ in $G_{Y}(f)$, there exists an l-equivalence $h: X^{\prime} \rightarrow X$ so that $f h \sim f^{\prime}$.

(3) Given a map $f^{\prime}: X \rightarrow Y^{\prime}$ in $G^{X}(f)$, there exists an l-equivalence $k: Y^{\prime} \rightarrow Y$ so that $k f^{\prime} \sim f$.

(b) If $Y$ is an $H$-space and $X=S X^{\prime \prime}$ then:

(1) Given two spaces $X^{\prime}, Y^{\prime}$, a function $f^{\prime}: S X^{\prime} \rightarrow Y^{\prime}$ and homotopy equivalences $h_{p_{i}}:\left(S X^{\prime}\right)_{p_{i}} \rightarrow X_{p_{i}}, k_{p_{i}}: Y_{p_{i}}^{\prime} \rightarrow Y_{p_{i}}$ satisfying $f_{p_{i}} h_{p_{i}} \sim k_{p_{i}} f_{p_{i}}^{\prime}(i=1, \ldots, s)$, there exist l-equivalences $h: S X^{\prime} \rightarrow X, k: Y^{\prime} \rightarrow Y$ so that $f h \sim k f^{\prime}$.

(2) Given a map $f: S X^{\prime} \rightarrow Y$ in $G_{Y}(f)$, there exists an l-equivalence $h: S X^{\prime} \rightarrow X$ so that $f h \sim f^{\prime}$.

(3) Given a map $f^{\prime}: X \rightarrow Y^{\prime}$ in $G^{X}(f)$, there exists an l-equivalence $k: Y^{\prime} \rightarrow Y$ so that $k f^{\prime} \sim f$.

(c) If $X=S^{n}$ and $Y=S^{2 m-1}$ then:

(1) Given a function $f^{\prime}: X \rightarrow Y$ and homotopy equivalences $h_{p_{i}}: X_{p_{i}} \rightarrow X_{p_{i}}, k_{p_{i}}$ : $Y_{p_{i}} \rightarrow Y_{p_{i}}$ satisfying $f_{p_{i}} h_{p_{i}} \sim k_{p_{i}} f_{p_{i}}^{\prime}(i=1, \ldots, s)$ there exist l-equivalences $h: X \rightarrow X$, $k: Y \rightarrow Y$ so that $f h \sim k f^{\prime}$.

(2) Given a map $f^{\prime}: X \rightarrow Y^{\prime}$ in $G_{Y}(f)$ there exists an l-equivalence $h: X \rightarrow X$ so that $f h \sim f^{\prime}$. 
Proof. (a) (1) Since $X$ and $Y$ are $H$-spaces, by 2.1, for every $i$ there exist integers $n_{1, i}, n_{2, i},\left(n_{1, i}, p_{i}\right)=\left(n_{2, i}, p_{i}\right)=1$, so that $\phi_{n_{1, i}} \circ h_{p_{i}}$ and $\phi_{n_{2, i}} \circ k_{p_{i}}$ are induced by functions $h_{i}^{\prime}: X^{\prime} \rightarrow X$ and $k_{i}^{\prime}: Y^{\prime} \rightarrow Y$.

As $f$ is an $H$-map, for every $i$, the $p$-localization of $f\left(\phi_{n_{2, i}} \circ h_{i}^{\prime}\right)$ and $\left(\phi_{n_{1, i}} \circ k_{i}^{\prime}\right) f^{\prime}$ are homotopic:

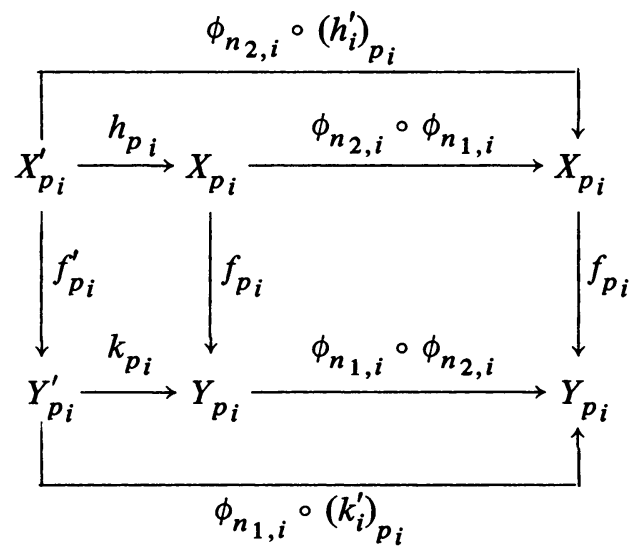

Hence, there exist integers $n_{i},\left(n_{i}, p_{i}\right)=1$, so that

$$
\left(\phi_{n_{i}} \phi_{n_{1, i}} k_{i}^{\prime}\right) f^{\prime} \sim \phi_{n_{i}}\left(\phi_{n_{1, i}} k_{i}^{\prime}\right) f^{\prime} \sim \phi_{n_{i}} f\left(\phi_{n_{2, i}} h_{i}^{\prime}\right) \sim f\left(\phi_{n_{i}} \phi_{n_{2, i}} h_{i}^{\prime}\right)
$$

Define $m=\prod_{i=1}^{s} p_{i}, h_{i}^{\prime \prime}=\phi_{m / p_{i}} \phi_{n_{1}, n_{2,}} h_{i}^{\prime}, k_{i}^{\prime \prime}=\phi_{n / p_{i}} \phi_{n_{i} n_{1, i}} k_{i}^{\prime}$. Then $h=\sum_{i=1}^{s} h_{i}^{\prime \prime}$ and $k=\sum_{i=1}^{s} k_{i}^{\prime \prime}$ are the desired maps. Indeed since $\pi_{*}(h) \otimes Z_{p_{i_{0}}}=\Sigma_{i} \pi_{*}\left(h_{i}^{\prime \prime}\right) \otimes Z_{p_{i_{0}}}=$ $\pi_{*}\left(h_{i_{0}}^{\prime \prime}\right) \otimes Z_{p_{i_{0}}}$ and $h_{i_{0}}^{\prime \prime}$ is a $p_{i_{0}}$-equivalence, $h$ is an $l$-equivalence. Similarly one gets that $k$ is an $l$-equivalence. It is clear that $f h \sim k f^{\prime}$.

(2) Since for every $p_{i}$ there exists a homotopy equivalence $h_{p_{i}}: X_{p_{i}}^{\prime} \rightarrow X_{p_{i}}$ satisfying $f_{p_{i}} h_{p_{i}} \sim f_{p_{i}}^{\prime}=1_{Y_{p_{i}}} f_{p_{i}}^{\prime}$, it follows from (1) that there exists an integer $n,(n, l)=1$, and an $l$-equivalence $h^{\prime}: X^{\prime} \rightarrow X$ so that $f h^{\prime} \sim \phi_{n} f^{\prime}$.

Assume that $n=q_{1}^{r_{1}} \ldots q_{v}^{r_{0}}$ where every $q_{i}$ is a prime. Since for every $q_{i}$ there exists a homotopy equivalence $h_{q_{i}}: X_{q_{i}}^{\prime} \rightarrow X_{q_{i}}$ satisfying $f_{q_{i}} h_{q_{i}} \sim f_{q_{i}}^{\prime}$, it follows from (1) that there exist an integer $m,(m, n)=1$, and an $l$-equivalence $h^{\prime \prime}: X^{\prime} \rightarrow X$ so that $f h^{\prime \prime} \sim \phi_{m} f^{\prime}$.

Let $a$ and $b$ be integers satisfying $a n+b l m=1$. Define $h: X^{\prime} \rightarrow X$ by $h=\phi_{a} h^{\prime}$ $+\phi_{b l} h^{\prime \prime}$. Since $\pi_{*}(h) \otimes Z_{l}=\pi_{*}\left(\phi_{a} h^{\prime}\right) \otimes Z_{l}$ and $h^{\prime}$ is an $l$-equivalence, $h$ is an l-equivalence. But $f h=f\left(\phi_{a} h^{\prime}+\phi_{b l} h^{\prime \prime}\right) \sim \phi_{a} f h^{\prime}+\phi_{b l} f h^{\prime \prime} \sim \phi_{a} \phi_{n} f^{\prime}+\phi_{b l} \phi_{m} f^{\prime} \sim$ $\phi_{a n+b l m} f^{\prime} \sim f^{\prime}$; hence $h$ is the desired map.

(3) is proved similarly.

(b) (1) Since $Y$ is an $H$-space and $\left(S X^{\prime}\right)_{p_{i}}=S X_{p_{i}}^{\prime}$, there exist integers $n_{1, i}, n_{2, i}$, $\left(n_{1, i}, p_{i}\right)=\left(n_{2, i}, p_{i}\right)=1$, so that the maps $h_{p_{i}} \eta_{n_{1, i}}$ and $\phi_{n_{2, i}} k_{p_{i}}$ are induced by maps $h_{i}^{\prime}$ : $S X^{\prime} \rightarrow X$ and $k_{i}^{\prime}: Y^{\prime} \sim Y$. 
Consider the following diagram:

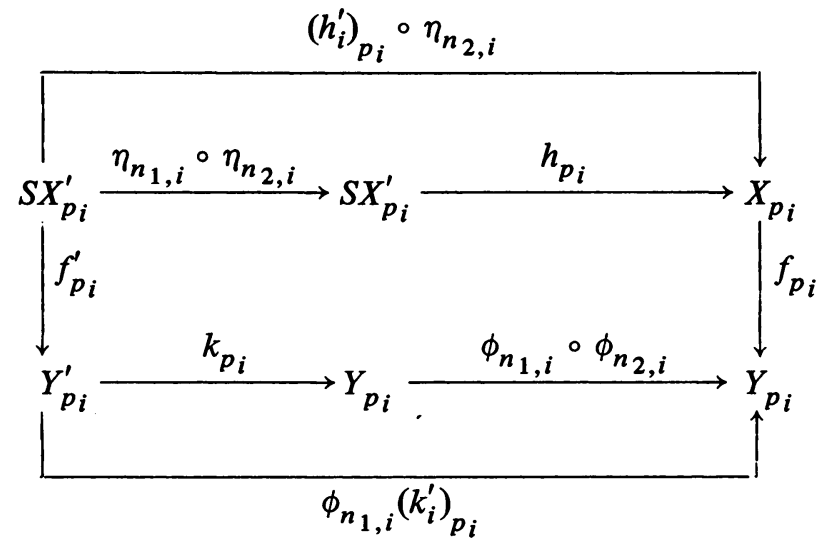

Since $f_{p_{i}} h_{p_{i}} \eta_{n_{1, i}} \eta_{n_{2, i}} \sim \phi_{n_{1, i}} \phi_{n_{2, i}} k_{p_{i}} f_{p_{i}}^{\prime}$, for every $i$ the $p_{i}$-localization of $f\left(h_{i}^{\prime} \eta_{n_{2, i}}\right)$ and $\left(\phi_{n_{1, i}} k_{i}^{\prime}\right) f^{\prime}$ are homotopic. Therefore there exist integers $n_{i},\left(n_{i}, p_{i}\right)=1$, so that

$$
\left(\phi_{n_{i}} \phi_{n_{1, i}} k_{i}^{\prime}\right) f^{\prime} \sim \phi_{n_{i}}\left(\phi_{n_{1, i}} k_{i}^{\prime}\right) f^{\prime} \sim \phi_{n_{i}}\left(f h_{i}^{\prime} \eta_{n_{2, i}}\right) \sim f\left(h_{i}^{\prime} \eta_{n_{2, i}} \eta_{n_{i}}\right)
$$

Define $m=\prod_{i=1}^{s} p_{i}$,

$$
h_{i}^{\prime \prime}=h_{i}^{\prime} \eta_{n_{2, i} n_{i}} \eta_{m / p_{i}}, \quad k_{i}^{\prime}=\phi_{m / p_{i}} \phi_{n_{i} n_{1, i}} k_{i}^{\prime}
$$

Then $h=\Sigma_{i} h_{i}^{\prime \prime}$ and $k=\Sigma_{i} k_{i}^{\prime \prime}$ are the desired maps: $h$ and $k$ are obviously $l$-equivalences and $f h \sim k f^{\prime}$.

(2) and (3) follow from (1) in the same way that (2) and (3) of part (a) follow from (1) of part (a).

(c) (1) Choose localizations $\varphi_{i}: S^{n} \rightarrow S_{p_{i}}^{n}, \psi_{i}: S^{2 m-1} \rightarrow S_{p_{i}-1}^{2 m-1}$ so that the following diagram is commutative:

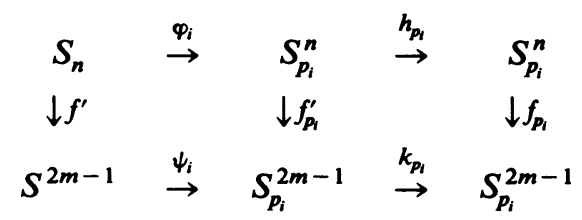

Since $h_{p_{i}} \varphi_{i} \in\left(\pi_{n} S^{n}\right)_{p_{i}}$ and $k_{p_{i}} \psi_{i} \in\left(\pi_{2 m-1} S^{2 m-1}\right)_{p_{i}}$, for every $i$, there exists an integer $v_{i},\left(v_{i}, p_{i}\right)=1$, so that $h_{p_{i}} \varphi_{i} \eta_{v_{i}}$ and $k_{p_{i}} \psi_{i} \eta_{v_{i}}$ are induced by maps $\tilde{h}_{i}: S^{n} \rightarrow S^{n}$ and $\tilde{k}_{i}: S^{2 m-1} \rightarrow S^{2 m-1}$. 
Consider the following diagram:

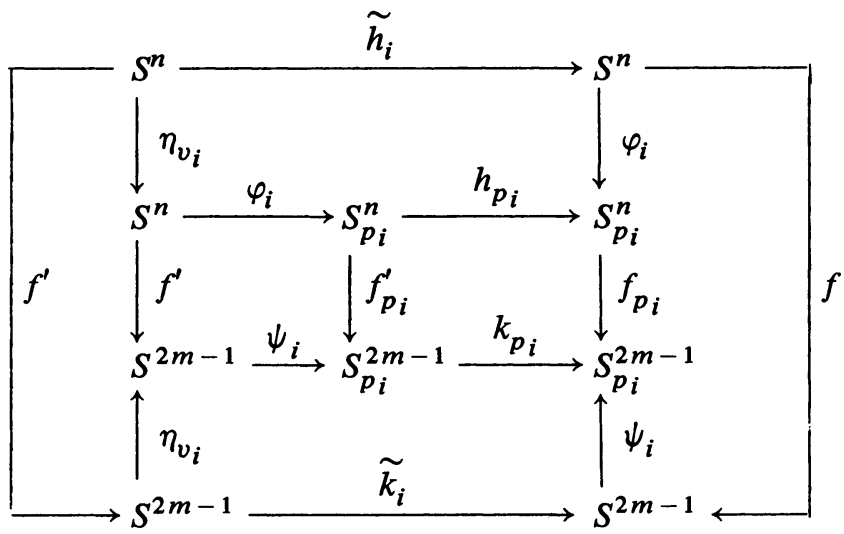

For $n=2 m-1$ the diagram commutes; hence in this case $f_{p_{i}}\left(\tilde{h_{i}}\right)_{p_{i}} \sim\left(\tilde{k_{i}}\right)_{p_{i}} f_{p_{i}}^{\prime}$. For $n>2 m-1$ the two squares and all the rectangles except the left one are commutative. Therefore in order to prove the existence of $p_{i}$-equivalences, $h_{i}^{\prime}: S^{n} \rightarrow S^{n}$ and $k_{i}^{\prime}: S^{2 m-1} \rightarrow S^{2 m-1}$ so that $f_{p_{i}}\left(h_{i}^{\prime}\right)_{p_{i}} \sim\left(k_{i}^{\prime}\right) f_{p_{i}}^{\prime}$, it suffices to prove

2.1.1. LemMA. For every map $f^{\prime}: S^{n} \rightarrow S^{2 m-1}$ and every integer $v$, there exists an integer $t$ so that $\eta_{v} f^{\prime} \sim f^{\prime} \eta_{v}$.

Proof of 2.1.1. Let $f_{1}, f_{2}: S^{n} \rightarrow S^{2 m-1}$ be arbitrary maps. Consider the diagram:

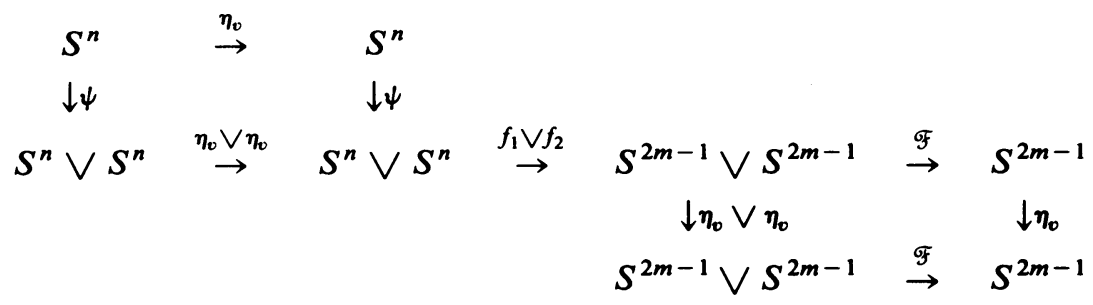

Obviously the right-hand square is commutative. Considering the homology homomorphisms one can easily see that the left-hand square is commutative as well. Hence $\eta_{v}\left(f_{1}+f_{2}\right) \sim \eta_{v} f_{1}+\eta_{v} f_{2}$ and $\left(f_{1}+f_{2}\right) \eta_{v} \sim f_{1} \eta_{v}+f_{2} \eta_{v}$.

Since the order of $f^{\prime}: S^{n} \rightarrow S^{2 m-1}$ is finite there exist $f_{1}, f_{2}: S^{n} \rightarrow S^{2 m-1}$ satisfying $f^{\prime}=f_{1}+f_{2},\left(\left|f_{1}\right|, v\right)=1$ and $\left|f_{2}\right| / v^{a}$ (for some integer $a$ ). Hence $f_{2} \eta_{v^{a}}$ $\sim *$. As for every $p_{j}, p_{j} / v, H^{*}\left(\eta_{v}, Z_{p_{j}}\right)=0$, there exists an integer $b$ so that, for every $p_{j} / v, \pi_{*}\left(\eta_{v^{b}}, Z_{p_{j}}\right)=0$ (Zabrodsky [9, proof of Theorem 4.1.4]). Hence for every $p_{j} / v, 0=\left(\eta_{v^{b}}\right)_{*}: \pi_{*} S^{2 m-1} \otimes Z_{p_{j}} \rightarrow \pi_{*} S^{2 m-1} \otimes Z_{p_{j}}$. Consequently there exists an integer $c$ so that $0=\left(\eta_{v} b c\right):\left.f_{2}\right|_{\pi_{*}} S^{2 m-1} \rightarrow\left|f_{2}\right|_{\pi_{*}} S^{2 m-1}$. Define $d=a b c$. Obviously for every $d^{\prime}>d,\left(f_{1}+f_{2}\right) \eta_{v^{d^{\prime}}} \sim f_{1} \eta_{v^{d^{\prime}}}$ and $\eta_{v^{d^{d}}}\left(f_{1}+f_{2}\right) \sim \eta_{v} d^{d} \cdot f_{1}$.

Since $\left(\left|f_{1}\right|, v^{d}\right)=1$ there exists an integer $\tilde{d}>d$ so that $v^{d} \equiv 1\left(\left|f_{1}\right|\right)$. Consider the map $\eta_{v} \dot{d}: S^{2 m-1} \rightarrow S^{2 m-1}$. This map is an $l$-equivalence; hence there exists an integer $e$ so that $1=\eta_{\left(v^{j}\right)^{e}}:\left|f_{1}\right| \pi_{n} S^{2 m-1} \rightarrow\left|f_{1}\right| \pi_{n} S^{2 m-1}$. Define $t=(\tilde{d})^{e}$. Since

$$
f^{\prime} \eta_{v^{\prime}}=\left(f_{1}+f_{2}\right) \eta_{v^{\prime}} \sim f_{1} \eta_{v^{\prime}} \sim f_{1} \sim \eta_{v} f_{1} \sim \eta_{v^{\prime}}\left(f_{1}+f_{2}\right)=\eta_{v} f^{\prime},
$$

$t$ is the desired integer which completes the proof of 2.1.1. 
Let $h_{i}^{\prime}: S^{n} \rightarrow S^{n}, k_{i}^{\prime}: S^{2 m-1} \rightarrow S^{2 m-1}$ be $p_{i}$-equivalences satisfying $f_{p_{i}}\left(h_{i}^{\prime}\right)_{p_{i}} \sim$ $\left(k_{i}^{\prime}\right)_{p_{i}} f_{p_{i}}^{\prime}$. As $k_{i}^{\prime} f^{\prime}, f h_{i}^{\prime} \in \pi_{n} S^{2 m-1}$ there exist integers $u_{i},\left(u_{i}, p_{i}\right)=1$, so that $f h_{i}^{\prime} \eta_{u_{i}} \sim$ $k_{i}^{\prime} f^{\prime} \eta_{u_{i}}\left(\eta_{u_{i}}: S^{n} \rightarrow S^{n}\right)$. Consequently we obtain from 2.1.1 that there exist integers $w_{i}$ satisfying

$$
f h_{i}^{\prime} \eta_{\mu_{i}^{w_{i}}} \sim k_{i}^{\prime} f^{\prime} \eta_{\mu_{i}^{w_{i}}} \sim\left(k_{i}^{\prime} \eta_{\mu_{i}^{w_{i}}}\right) f^{\prime}
$$

Suppose that $\eta_{p_{i}} f^{\prime} \sim k_{i}^{\prime} f^{\prime} \eta_{p_{i}^{\prime}}, \eta_{2^{\prime}} f^{\prime} \sim f^{\prime} \eta_{2^{\prime}}$ and $p_{i} \neq 2$ for $i>1$. Define

$$
\begin{aligned}
& v=2^{t} \prod p_{i}^{r_{1}}
\end{aligned}
$$

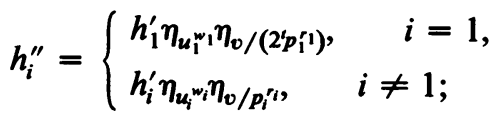

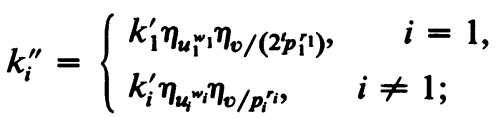

$$
\begin{aligned}
& h=\sum_{i} h_{i}^{\prime \prime}, \quad k=\sum_{i} k_{i}^{\prime \prime} .
\end{aligned}
$$

Obviously $h$ and $k$ are $l$-equivalences. In order to prove that $f h \sim k f^{\prime}$ it is enough to prove that $\left(\Sigma_{i} k_{i}^{\prime \prime}\right) f^{\prime} \sim \Sigma\left(k_{i}^{\prime \prime} f^{\prime}\right)$.

Let $\iota_{j}: S^{2 m-1} \rightarrow S^{2 m-1} \times\left(S^{2 m-1}\right)^{s-1}$ be the inclusion into the $j$ th factor. There exists a map $\alpha: S^{2 m-1} \times\left(S^{2 m-1}\right)^{s-1} \rightarrow S^{2 m-1}$ so that for every $j>1$ the diagram

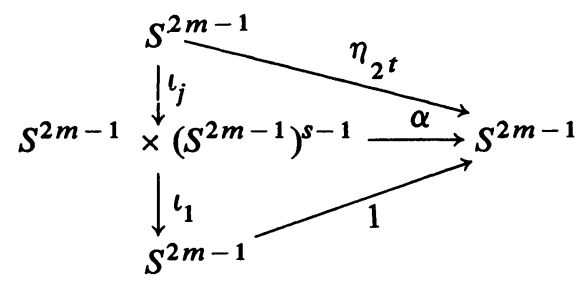

is commutative. Therefore the following diagram is commutative:

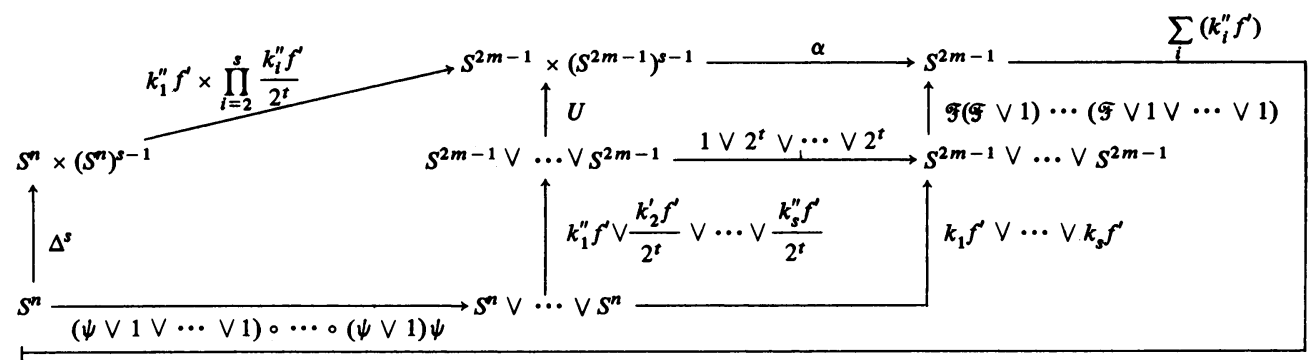

$$
\left(\Delta^{s}(x)=(x, \ldots, x)\right), \text { namely } \sum_{i}\left(k_{i}^{\prime \prime} f^{\prime}\right)=\alpha \circ\left(k_{1}^{\prime \prime} f^{\prime} \times \prod k_{i}^{\prime \prime} f^{\prime} / 2^{l}\right) \circ \Delta^{s} .
$$


Consequently the commutativity of the diagram

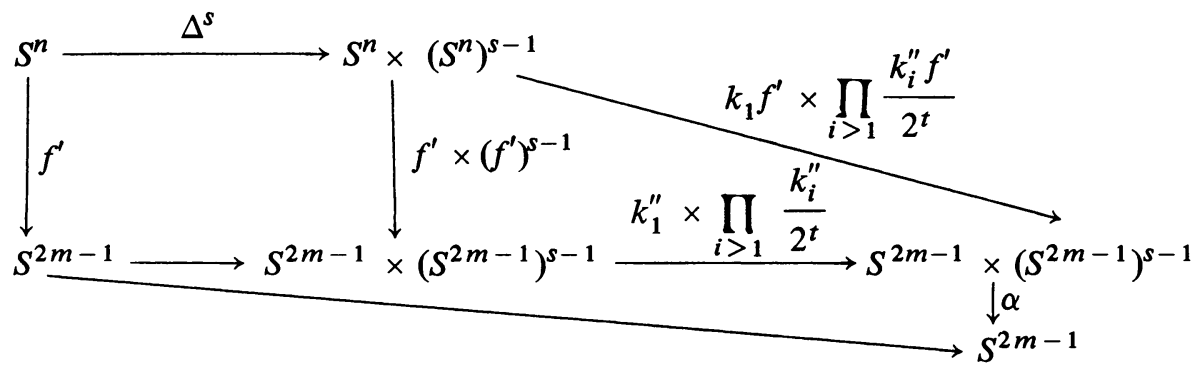

implies that $\left(\Sigma_{i} k_{i}^{\prime \prime}\right) f^{\prime} \sim \Sigma_{i}\left(k_{i}^{\prime \prime} f^{\prime}\right)$.

Since for any two maps $g_{1}, g_{2}: S^{n} \rightarrow S^{n}, f\left(g_{1}+g_{2}\right) \sim f g_{1}+f g_{2}$, (2) follows from (1) in the same way that (2) of part (a) follows from (1) of the same part.

Notation. Let $X$ be an $H_{0}$-space, denote by $N(X)$ the least integer satisfying either, for every $n>N(X), \pi_{n} X=0$ or, for every $n>N(X), H_{n} X=0$. (Recall that we consider only spaces with $N(X)<\infty$.)

2.3. Corollary. (a) Given an $H$-fibration $F \rightarrow X \stackrel{f}{\rightarrow} Y$; if $H^{*}(f, Q)$ is surjective then $G_{Y}(f)=0$.

(b) Given a fibration $F \rightarrow X \stackrel{f}{\rightarrow} Y$ so that $Y$ is an $H$-space and $X$ is an $H_{0}$-space: If $H^{*}(f, Q)$ is injective then $G^{X}(f)=0$.

(c) (a) and (b) hold also for a fibration of the form $F \rightarrow S X \rightarrow Y$ where $Y$ is an H-space.

Proof. (a) Let $F \rightarrow X \stackrel{f}{\rightarrow} Y$ be an $H$-fibration and let $l=\Pi_{n<N} \mid$ torsion $\pi_{n} X \mid \cdot$ |torsion $\pi_{n} F \mid$ where $N=\max \{N(X), N(Y)\}$. By Theorem 2.2 there exist $l$-equivalences $h: X^{\prime} \rightarrow X$ and $h^{\prime}: F \rightarrow F$ so that the following diagram commutes

$$
\begin{aligned}
F^{\prime} & \rightarrow X^{\prime} f^{\prime} \\
\downarrow h^{\prime} & \\
F & \rightarrow h{ }^{\prime} Y
\end{aligned}
$$

We shall prove that $h$ is a homotopy equivalence.

As $h_{\sharp}:{ }^{t} \pi_{*} X^{\prime} \rightarrow{ }^{t} \pi_{*} X$ and $h_{\sharp}^{\prime}:{ }^{t} \pi_{*} F^{\prime} \rightarrow^{t} \pi_{*} F\left({ }^{t} \pi_{*} X=\right.$ torsion $\left.\left(\pi_{*} X\right)\right)$ are isomorphisms and as $h, h^{\prime}$ are 0-equivalences, $h_{\sharp}: \pi_{*} X^{\prime} /$ torsion $\rightarrow \pi_{*} X /$ torsion and $h_{\sharp}^{\prime}$ : $\pi_{*} F^{\prime} /$ torsion $\rightarrow \pi_{*} F /$ torsion are monomorphisms, so are $h_{\sharp}: \pi_{*} X^{\prime} \rightarrow \pi_{*} X$ and $h_{\sharp}^{\prime}$ : $\pi_{*} F^{\prime} \rightarrow \pi_{*} F$ and $h$ is a homotopy equivalence if and only if $h_{\sharp}$ is a surjection.

Consider the following diagram:

$$
\begin{array}{ccccc}
\pi_{n} X^{\prime} & \stackrel{f_{\sharp}^{\prime}}{\rightarrow} & \pi_{n} Y & \stackrel{\partial^{\prime}}{\rightarrow} & \pi_{n-1} F^{\prime} \\
\downarrow h_{\sharp} & & \| & & \downarrow h_{\sharp}^{\prime} \\
\pi_{n} X & \stackrel{f_{\sharp}}{\rightarrow} & \pi_{n} Y & \stackrel{\partial}{\rightarrow} & \pi_{n-1} F
\end{array}
$$


Let $v \in \pi_{n} X$ be of infinite order. As $H^{*}(f, Q)$ is surjective, $H_{*}(f, Q)$ and $\pi_{*}(f) \otimes$ $Q$ are injective and so is $\pi_{*}(f) /$ torsion; hence $w=f_{\sharp} v$ is of infinite order as well. $0=\partial w=h_{\sharp}^{\prime} \partial^{\prime} w$; hence $\partial^{\prime}(w)=0$ and there exists $v^{\prime} \in \pi_{n} X^{\prime}$ so that $f_{\sharp}^{\prime} v^{\prime}=w$. Hence, $v-h_{\sharp} v^{\prime} \in \operatorname{ker} f_{\sharp} \subseteq$ torsion $\pi_{n} X \subseteq \operatorname{im} h_{\sharp}, v \in \operatorname{im} h_{\sharp}$ and $h_{\sharp}$ is surjective.

(b) and (c) are proved similarly.

3. The structure of $G(f), G^{X}(f)$ and $G_{Y}(f)$. In this section we use Zabrodsky's method of constructing the genus of an $H_{0}$-space (with a finite number of homotopy or homology groups-Zabrodsky [8]) to obtain elements in the genus of a map $f: X \rightarrow Y$ where $X$ and $Y$ are $H_{0}$-spaces. We go on to prove that every element in the genus of a map which satisfies the conditions of Theorem $I$ is obtained in this way. The same method is also good for constructing $G^{X}(f),\left(G_{Y}(f)\right)$ for maps which satisfy the conditions of Theorem III (I).

3.1. Definitions AND NOTATIONS. Let $P$ be the set of all primes. For any integer $t$ denote by $P_{t}$ the set of all primes which divide $t$ and by $\bar{t}$ the set $P-P_{t}$.

Let $X$ be an $H_{0}$-space, i.e. $H^{*}(X, Q)$ is a free commutative graded algebra. Denote by $[X, X]_{t}$ the set of homotopy classes of $t$-equivalences $f: X \rightarrow X$. Denote by $t(X)$ the number $\left.\Pi_{n<N(X)}\right|^{t} H^{n}(X) \mid$, by $K(X)$ the space $K\left(Q H^{*}(X, Z) /\right.$ torsion) and by $l(x)$ the number of integers $n$ for which $Q H^{n}(X, Q) \neq 0$. Let $\Gamma$ be a splitting $\operatorname{Hom}^{\circ}\left(Q H^{*}(X, Z) /\right.$ torsion, $Q H^{*}(X, Z) /$ torsion $) \rightarrow[K(X), K(X)]$ : $Q H^{*}(\Gamma(f), Z) /$ torsion $=f$.

Let $A$ be an $n \times n$ matrix. We shall say that $A$ is diagonal if

$$
A_{i j}= \begin{cases}\lambda_{i}, & i=j, i \leqslant \min (m, n), \\ 0, & \text { otherwise }\end{cases}
$$

(some of the $\lambda_{i}$ 's may be zero).

Suppose $f: X \rightarrow Y$ is a map and $X$ and $Y$ are $H_{0}$-spaces. Let $B_{X}=$ $\left\{x_{m_{1}^{\prime}}, x_{m_{2}^{\prime}}, \ldots, x_{m_{i}^{\prime}}\right\}, \operatorname{dim} x_{m_{i}^{\prime}}=m_{i}^{\prime}, m_{i}^{\prime} \leqslant m_{i+1}^{\prime}$ and $B_{Y}=\left\{y_{n_{1}^{\prime}}, y_{n_{2}^{\prime}}, \ldots, y_{n_{3}^{\prime}}\right\}$, dim $y_{n_{i}^{\prime}}$ $=n_{i}^{\prime}, n_{i}^{\prime} \leqslant n_{i+1}^{\prime}$ be bases for $Q H^{*}(X, Z) /$ torsion and $Q H^{*}(Y, Z) /$ torsion in which $Q H^{*}(f, Z) /$ torsion is represented by a diagonal matrix $A$.

Assume that $Q H^{m_{j}}(Y, Q) \neq 0$ for $j=1, \ldots, l(X), m_{1}<m_{2}<\ldots<m_{l(X)}$, and that $Q H^{n_{k}}(Y, Q) \neq 0$ for $k=1, \ldots, l(Y), n_{1}<n_{2}<\ldots<n_{l(Y)}$. Obviously for every $1 \leqslant i \leqslant r$ there exists a $j(1 \leqslant j \leqslant l(X))$ so that $m_{i}^{\prime}=m_{j}$, and for every $1 \leqslant i \leqslant s$ there exists a $K(1 \leqslant k \leqslant l(Y))$ so that $n_{i}^{\prime}=n_{k}$.

Let $\tilde{t}=\left.\Pi_{n<N}\right|^{t} H^{n}(X, Z) \mid(N=\max \{N(X), N(Y)\})$ and let $\psi: X \rightarrow K(X), \varphi$ : $Y \rightarrow K(Y)$ be rational equivalences realizing $\left\{\tilde{t}_{m_{i}}, \ldots, \tilde{t}_{m_{m_{2}}}\right\}$ and $\left\{\tilde{y}_{n_{i}^{\prime}}, \ldots, \tilde{t}_{n_{n^{\prime}}}\right\}$, respectively. Denote by $t$ the least common multiple of $\tilde{t},\left.\Pi_{n<N}\right|^{t} H^{n}(Y, Z) \mid$, $\Pi_{n<N} \mid \pi_{n}($ fiber $\varphi)\left|, \Pi_{n<N}\right| \pi_{n}$ (fiber $\left.\psi\right) \mid$ and the nonzero elements of $A$.

Denote by $D \subset Z^{l(X)+l(Y)}$ the set of pairs $\left(d, d^{\prime}\right), d=\left(d_{m_{1}}, \ldots, d_{m_{l(X)}}\right) \in Z^{l(X)}$, $d^{\prime}=\left(d_{n_{1}}^{\prime}, \ldots, d_{n_{l(Y)}^{\prime}}^{\prime}\right) \in Z^{l(Y)}$ satisfying the following conditions:

(a) For every $i,\left(d_{n_{i}}, t\right)=\left(d_{m_{i}}, t\right)=1$.

(b) If $Q H^{n_{i}}(f, Q)$ is a monomorphism and $Q H^{n_{i}}(Y, Q) \neq 0$ then $d_{n_{i}}^{\prime} / d_{n_{i}}$.

(c) If $Q H^{n_{i}}(f, Q)$ is an epimorphism and $Q H^{n_{1}}(X, Q) \neq 0$ then $d_{n_{i}} / d_{n_{i}}^{\prime}$.

(d) If $Q H^{n_{i}}(f, Q)$ is an isomorphism then $d_{n_{i}}=d_{n_{i}}^{\prime}$. 
3.2. TheOReM (ZABRodSKy [8]). Let $X$ be an $H_{0}$-space with $Q H^{m_{1}}(X, Q) \neq 0$ for $i=1, \ldots, l(X)$ and let $\psi: X \rightarrow K(X)$ be a rational equivalence. Suppose $t(X, \psi)$ is an integer divisible by $\left.\Pi_{n<N(X)}\right|^{t} \pi_{n}($ fiber $\psi) \mid$.

Then $G(X)$ admits an abelian group structure and there exists an exact sequence

$$
[X, X]_{t(X, \psi)} \stackrel{\alpha}{\rightarrow}\left[\left(Z_{t}^{*}(X, \psi)\right) / \pm 1\right]^{l(X)} \stackrel{\xi}{\rightarrow} G(X) \rightarrow 0
$$

where $\alpha$ is the composition

$$
[X, X]_{t(X, \psi)} \rightarrow \operatorname{aut}\left(Q H^{*}(X, Z) / \text { torsion } \otimes Z_{t(X, \psi)} \stackrel{\text { |det| }}{\rightarrow}\left[\left(Z_{t(X, \psi)}^{*}\right) / \pm 1\right]^{l(X)}\right.
$$

and $\xi$ is given as follows: Let $d_{1}, \ldots, d_{l(X)}$ be integers satisfying $\left(d_{i}, t(X, \psi)\right)=1$ for every $i$ and let $I_{d_{1}, \ldots, d_{l}(x)}: Q H^{*}(X, Z) /$ torsion $\rightarrow Q H^{*}(X, Z) /$ torsion satisfy $\operatorname{det}\left(I_{\left.d_{1}, \ldots, d_{k X}\right)} \mid Q H^{m}(X, Z) /\right.$ torsion $)=d_{i}$. Consider the following pull-back diagram

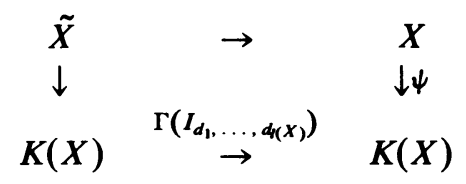

If $X$ has a finite number of homotopy groups define $\xi\left(d_{1}, \ldots, d_{l(X)}\right)=\tilde{X}$ and if $X$ is finite dimensional define $\xi\left(d_{1}, \ldots, d_{l(X)}\right)=H L_{\operatorname{dim} X}(\tilde{X})$.

3.3. Definition. Let $X$ be an $H_{0}$-space so that $Q H^{m}(X, Q) \neq 0$ for $i=$ $1, \ldots, l(X)$ and let $f: X \rightarrow X$ be a map. Suppose $d=\left(d_{1}, \ldots, d_{l(X)}\right) \in Z^{l(X)}$. We say that $f$ realizes $d$ if, for every $i, \operatorname{det}\left(Q H^{m}(f, Z) /\right.$ torsion $)=d_{i}$.

3.4. Proposition. Let $f: X \rightarrow Y$ be a map.

(a) If $X, Y$ are $H_{0}$-spaces, then for every pair $\left(d, d^{\prime}\right) \in D$ there exist a map $f^{\prime}$ : $X^{\prime} \rightarrow Y^{\prime}, G(f)$ and t-equivalences $h: X^{\prime} \rightarrow X$ and $k: Y^{\prime} \rightarrow Y$ so that $h$ realizes $d, k$ realizes $d^{\prime}$ and $f h \sim k f^{\prime}$.

(b) Let $f$ be an $H$-map and suppose $H^{*}(X, Q)$ and $H^{*}(Y, Q)$ are primitively generated. Then for every pair $\left(d, d^{\prime}\right) \in D$ there exist an $H$-map $f^{\prime}: X^{\prime} \rightarrow Y^{\prime}$ and $H$-maps $h$ and $k$ so that (a) is satisfied.

Proof. Let $B_{X}, B_{Y}, \varphi$ and $\psi$ be as in 3.1 and let $f: K(X) \rightarrow K(Y)$ satisfy $g \psi \sim \varphi f$.

Let $\alpha: K(X) \rightarrow K(X)$ and $\beta: K(Y) \rightarrow K(Y)$ satisfy:

$\alpha=\Pi \alpha_{i}\left(\alpha_{i}: K\left(Z, m_{i}^{\prime}\right) \rightarrow K\left(Z, m_{i}^{\prime}\right)\right), \operatorname{det}\left(Q H^{m_{1}}(\alpha, Z) /\right.$ torsion $)=d_{m_{i}}$.

$\beta=\Pi \beta_{i}\left(\beta_{i}: K\left(Z, n_{i}^{\prime}\right) \rightarrow K\left(Z, n_{i}^{\prime}\right)\right), \operatorname{det}\left(Q H^{n_{1}}(\beta, Z) /\right.$ torsion $)=d_{n_{i}}^{\prime}$.

$g \alpha \sim \beta g$ (such $\alpha$ and $\beta$ exist since, for every $i, g^{*}\left(l_{n^{\prime}}\right)=\lambda_{i} l_{n_{i}^{\prime}}$ or $g^{*}\left(l_{m^{\prime}}\right)=0$ ).

Consider the diagram

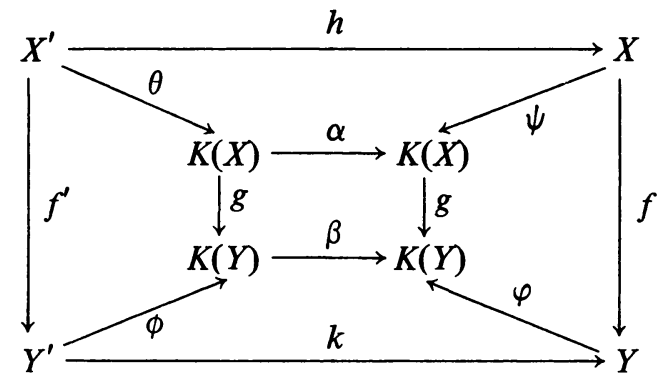


where $X^{\prime}$ is the pull-back of $X \stackrel{\psi}{\rightarrow} K(X) \stackrel{\alpha}{\leftarrow} K(X)$ and $Y^{\prime}$ is the pull-back of $Y \stackrel{\varphi}{\rightarrow} K(Y) \stackrel{\beta}{\leftarrow} K(Y)$.

Since the lower trapezoid is a pull-back and $\varphi f h \sim \beta g \theta$, there exists a map $f^{\prime}$ : $X^{\prime} \rightarrow Y^{\prime}$ so that $k f^{\prime} \sim f h$ and $\phi f^{\prime} \sim g \theta$. Consider the diagram:

$$
\begin{array}{ccccc}
X_{t}^{\prime} & \stackrel{\theta_{t}}{\rightarrow} & K(X)_{i} & \stackrel{\left(\Psi_{i}\right)^{-1}}{\rightarrow} & X_{\bar{t}} \\
\downarrow f_{t}^{\prime} & & \downarrow g_{i} & & \downarrow f_{\bar{t}} \\
Y_{t}^{\prime} & \stackrel{\phi_{i}^{-}}{\rightarrow} & K(Y)_{\bar{t}} & \stackrel{\left(\varphi_{i}^{-}\right)^{-1}}{\rightarrow} & Y_{\bar{t}}
\end{array}
$$

(If $f: X \rightarrow Y$ is a homotopy equivalence $f^{-1}$ denotes the homotopy inverse of $f$.) Since this diagram is commutative and its horizontal rows are homotopy equivalences the map $f^{\prime}: X^{\prime} \rightarrow Y^{\prime}$ belongs to $G(f)$.

(b) Choose bases $\left\{x_{m_{1}^{\prime}}, \ldots, x_{m_{r}^{\prime}}\right\},\left\{y_{n_{1}^{\prime}}, \ldots, y_{n_{r}^{\prime}}\right\}$ for $P H^{*}(X, Z) /$ torsion and $P H^{*}(Y, Z) /$ torsion, respectively, in which $P H^{*}(f, Z) /$ torsion is represented by a diagonal matrix. (By Curjel [2] such bases exist.) Let $\psi: X \rightarrow K(X), \varphi: Y \rightarrow K(Y)$ realize $\left\{\tilde{t} x_{m_{1}^{\prime}}, \ldots, \tilde{t} x_{m_{r}^{\prime}}\right\}$ and $\left\{\tilde{t}_{n_{1}^{\prime}}, \ldots, \tilde{t}_{n_{s}^{\prime}}\right\}$, respectively. Obviously $\psi$ and $\varphi$ are $H$-maps.

Let $g, \alpha$ and $\beta$ be as in part (a). Consider diagram 3.4.1. Obviously $h$ and $k$ are $H$-maps and $f^{\prime} \in G(f)$. We shall prove that $f^{\prime}$ is an $H$-map:

Since the maps

$$
\begin{aligned}
(\Omega \beta)_{*}:\left[X^{\prime} \times X^{\prime}, \Omega K(Y)\right] & \rightarrow\left[X^{\prime} \times X^{\prime}, \Omega K(Y)\right], \\
(\Omega \varphi)_{*}:\left[X^{\prime} \times X^{\prime}, \Omega Y\right] & \rightarrow\left[X^{\prime} \times X^{\prime}, \Omega K(Y)\right]
\end{aligned}
$$

are $t$ and $\bar{t}$ equivalences, respectively, the map

$$
\begin{array}{r}
(\Omega \beta)_{*}+(\Omega \varphi)_{*}:\left[X^{\prime} \times X^{\prime}, \Omega K(Y)\right] \oplus\left[X^{\prime} \times X^{\prime}, \Omega Y\right] \rightarrow\left[X^{\prime} \times X^{\prime}, \Omega K(Y)\right] \\
\left((\Omega \beta)_{*}+(\Omega \varphi)_{*}\right)(a, b)=(\Omega \beta)_{*}(a)+(\Omega \varphi)_{*}(b)
\end{array}
$$

is an epimorphism (Arkowitz [1, Proposition 4.3]). This together with the fact that $\phi f^{\prime}$ and $k f^{\prime}$ are $H$-maps implies (Arkowitz [1, Proposition 10.3]) that $f^{\prime}$ is an $H$-map.

3.5. Corollary. (a) If $(d, 1) \in D$ then Proposition 3.4 is true for $G_{Y}(f)$.

(b) If $\left(1, d^{\prime}\right) \in D$ then Proposition 3.4 is true for $G^{X}(f)$.

Proof. (a) Choose $\phi=\varphi, k=1, \beta=1$.

(b) Choose $\theta=\psi, h=1, \alpha=1$.

3.6. Proposition. Suppose $X_{1}, X_{2}$ are $H$-spaces so that $H^{*}\left(X_{i}, Q\right)(i=1,2)$ are primitively generated; $Y_{1}, Y_{2}$ are $H_{0}$-spaces; $f: X_{1} \rightarrow X_{2}$ an $H$-map, and $g: Y_{1} \rightarrow Y_{2}$ a map.

Let $B_{X_{i}}=\left\{x_{i_{1}}, \ldots, x_{i m_{i}}\right\}$ be bases for $P H^{*}\left(X_{i}, Z\right) /$ torsion, and let $B_{Y_{i}}=$ $\left\{y_{i}, \ldots, y_{i n_{i}}\right\}$ be bases for $H^{*}\left(Y_{i}, Z\right) /$ torsion. Denote by $A$ and $B$ the matrices of $P H^{*}(f, Z) /$ torsion and $H^{*}(g, Z) /$ torsion in these bases.

There exists an integer $t(f, g)$ depending on $X_{i}, Y_{i}, f$ and $g$ so that: Given a pair of matrices (over $Z)\left(C_{1}, C_{2}\right)$ satisfying $C_{1} A=B C_{2}$, there exist functions $h_{i}: Y_{i} \rightarrow X_{i}$ $(i=1,2)$ so that the following conditions are satisfied: 
(a) The matrix of $H^{*}\left(h_{i}, Z\right) /$ torsion $\mid\left(P H^{*}\left(X_{i}, Z\right) /\right.$ torsion) relative to the bases $B_{X_{i}}$ and $B_{Y_{i}}$ is $t(f, g) C_{i}$.

(b) $f h_{1} \sim h_{2} g$.

Proof. It is enough to prove the proposition in case that $B_{X_{i}}$ and $B_{Y_{i}}(i=1,2)$ are bases in which the matrices $A$ and $B$ are diagonal.

Let $\lambda$ be the multiple of the nonzero elements of $A$ and $B$ and let $t$ be as in 3.1.

Let $\mathcal{C}=\left\{\left(C_{1}, C_{2}\right)||\left(C_{1}\right)_{i j}|\leqslant t \lambda,|\left(C_{2}\right)_{i j} \mid \leqslant t \lambda\right.$ for every $i$ and $\left.j, C_{1} A=B C_{2}\right\}$. To each pair $\left(C_{1}, C_{2}\right) \in \mathcal{C}$ correspond functions $h_{i}: Y_{i} \rightarrow X_{i}$ the matrices of which relative to $B_{X_{i}}$ and $B_{Y_{i}}$ are $\lambda t C_{i}$ (Zabrodsky [8, Proposition 1.8]). Since $X_{i}$ and $Y_{i}$ are $\bar{t}$-equivalent to $K\left(X_{i}\right)$ and $K\left(Y_{i}\right)$, respectively, the $\bar{t}$-localizations of $f h_{1}$ and $h_{2} g$ coincide. Hence there exists an integer $s_{\left(C_{1}, C_{2}\right)}$ so that $\left(\phi_{s_{\left(c_{1}, c_{2}\right)}} h_{2}\right) g \sim f\left(\phi_{s_{\left(C_{1}, c_{2}\right)}} h_{1}\right)$. This together with the finiteness of the set $\mathcal{C}$ implies the existence of an integer $s$ which is good for every pair $\left(C_{1}, C_{2}\right) \in \mathcal{C}$. We shall prove that $s=t(f, g)$.

As $A$ and $B$ are diagonal and $C_{1} A=B C_{2}$, for every $i$ and $j,\left(C_{1}\right)_{i j} a_{i j}=b_{i i}\left(C_{2}\right)_{i j}$. If $b_{i i}=b \cdot b^{\prime}$ where $b /\left(C_{1}\right)_{i j}$ and $b^{\prime} / a_{i j}$ then

$$
\left[\left(C_{1}\right)_{i j} / b\right] \cdot b a_{j j}=\left(C_{1}\right)_{i j} a_{j j}=b_{i i}\left(C_{2}\right)_{i j}=b a_{i j}\left[\left(C_{2}\right)_{i j} /\left(a_{i j} / b^{\prime}\right)\right] .
$$

Assume that $\left[\left(C_{1}\right)_{i j} / b\right]=t l_{i j}+c_{i j}$ where $\left|c_{i j}\right|<t$ or $c_{i j}=0$. For every $1<k<$ $\max \left\{l_{i j}\right\}+1$ define matrices $C_{1}^{k}, C_{2}^{k}$ as follows:

$$
\left(C_{1}^{k}\right)_{i j}=\left\{\begin{array}{ll}
c_{i j} b, & k=1, \\
t b, & 1<k \leqslant l_{i j}+1, \\
0, & k>l_{i j}+1,
\end{array} \quad\left(C_{2}^{k}\right)_{i j}= \begin{cases}c_{i j}\left[a_{i j} / b^{\prime}\right], & k=1, \\
t \cdot\left[a_{i j} / b^{\prime}\right], & 1<k<l_{i j}+1, \\
0, & k>l_{i j}+1 .\end{cases}\right.
$$

Obviously, for every $k$, the pair $\left(C_{1}^{k}, C_{2}^{k}\right) \in \mathcal{C}$ and $\left(C_{1}, C_{2}\right)=\Sigma_{k}\left(C_{1}^{k}, C_{2}^{k}\right)$.

Let $h_{i}^{k}: Y_{i} \rightarrow X_{i}(i=1,2)$ be maps the matrices of which are $s C_{i}^{k}$ and which satisfy $f h_{1}^{k} \sim h_{2}^{k} g$. Since $B_{X_{i}}$ are bases for $P H^{*}\left(X_{i}, Z\right) /$ torsion and $f$ is an $H$-map, the matrices of $\Sigma_{k} h_{1}^{k}$ (relative to $B_{X_{i}}$ and $B_{Y_{i}}$ ) are $s C_{i}$ and $f\left(\Sigma_{k} h_{1}^{k}\right) \sim\left(\Sigma h_{2}^{k}\right) g$. Consequently $t(f, g)=s$.

3.7. Remark. If $C_{1} A=B C_{2}$ and $C_{1}=0\left(C_{2}=0\right)$ we obtain that

$$
* \sim h_{2} g\left(f h_{1} \sim *\right) \text {. }
$$

3.8. Corollary. Proposition 3.6 remains true if we substitute $X_{1}=Y_{1}=$ $\bigvee_{\text {finite }} S^{m_{j}}, f: X_{1} \rightarrow X_{2}$ a function and replace $B_{X_{1}}$ and $B_{Y_{1}}$ by bases $B_{X_{1}}^{\prime}$ and $B_{Y_{1}}^{\prime}$ of $H^{*}\left(\bigvee S^{m_{3}}\right)$.

Proof. Analogous to the proof of Theorem 3.6, since if $k_{1}, k_{2}: Y_{1} \rightarrow X_{1}$ are functions the matrices of which (relative to the bases $B_{X_{1}}^{\prime}$ and $B_{Y_{1}}^{\prime}$ ) are $C_{1}$ and $C_{2}$ and $l_{1}, l_{2}: Y_{2} \rightarrow X_{2}$ are functions which satisfy $f k_{1} \sim l_{1} g, f k_{2} \sim l_{2} g$. Then the matrix of $k_{1}+k_{2}$ is $C_{1}+C_{2}$ and $f\left(k_{1}+k_{2}\right) \sim\left(l_{1}+l_{2}\right) g$.

3.9. Corollary. If in Proposition $3.6 Y_{2}=X_{2}, B_{Y_{2}}=B_{X_{2}}$ we obtain that there exists an integer $t(f, g)$ depending on $X_{1}, Y_{1}, X_{2}, f$ and $g$, so that for every matrix $C$ which satisfies $C A=B$, there exists a function $h: Y_{1} \rightarrow X_{1}$ so that:

(a) The matrix of $H^{*}(h, Z) /$ torsion $\mid\left(P H^{*}\left(X_{1}, Z\right) /\right.$ torsion) relative to the bases $B_{X_{1}}$ and $B_{Y_{1}}$ is $t(f, g) C$.

(b) $f h \sim \phi_{t(f, g)} g$. 
Proof. It is enough to prove the assertion in the case that $B_{X_{i}}(i=1,2)$ are bases in which the matrix $A$ of $P H^{*}(f, Z) /$ torsion is diagonal.

Since $C A=B$, the matrix $C$ is of the form

$$
C=\left(\begin{array}{ll}
C_{1} & C_{2} \\
C_{3} & C_{4}
\end{array}\right)
$$

where $C_{1}$ is completely determined by $A$ and $B$. The corollary follows from the fact that every matrix $D$ of the form

$$
D=\left(\begin{array}{cc}
0 & C_{2} \\
C_{3} & C_{4}
\end{array}\right)
$$

can be written as $D=\sum_{k \text { finite }} D_{k}$, where $\left|\left(D_{k}\right)_{i j}\right|<t$ for every $i$ and $j$ and $D_{k} A=0$ for every $k$.

3.10. Corollary. If in Proposition 3.6 $Y_{1}=X_{1}, B_{X_{1}}=B_{Y_{1}}=a$ basis for $H^{*}\left(Y_{1}, Z\right) /$ torsion, we obtain that there exists an integer $t(f, g)$ so that for every matrix $C$ which satisfies $A=B C$ there exists a map $k: Y_{2} \rightarrow X_{2}$ so that:

(a) The matrix of $H^{*}(k, Z) /$ torsion $\mid\left(P H^{*}(X, Z) /\right.$ torsion) relative to the bases $B_{X_{2}}$ and $B_{Y_{2}}$ is $t(f, g) C$.

(b) $k g \sim \phi_{t(f, g)} f$.

Proof. Similar to the proof of 3.9.

REMARK. The corollary remains true if one replaces the conditions that $X_{1}$ is an $H$-space and $f$ is an $H$-map by the conditions that $X_{1}$ is an $H_{0}$-space and $f$ is a map.

If $A$ and $B$ are matrices denote by $A * B$ the matrix $\left(\begin{array}{ll}A & 0 \\ 0 & B\end{array}\right)$.

If $X, Y$ are $H_{0}$-spaces and for every $1<i \leqslant l$ either $H^{s_{1}}(X, Q) \neq 0$ or $H^{s_{1}}(Y, Q)$ $\neq 0$ we can identify

$\operatorname{Hom}_{Z}^{0}\left(Q H^{*}(Y, Z) /\right.$ torsion, $Q H^{*}(X, Z) /$ torsion) with the set of matrices $A^{Q}=$ $A_{s_{1}}^{Q} * A_{s_{2}}^{Q} * \cdots * A_{s_{1}}^{Q}$,

$\operatorname{Hom}_{Z}^{0}\left(D H^{*}(Y, Z) /\right.$ torsion, $D H^{*}(X, Z) /$ torsion) with the set of matrices $A^{D}=$ $A_{s_{1}}^{D} * A_{s_{2}}^{D} * \cdots * A_{s_{l}}^{D}$ and

$\operatorname{Hom}_{Z}^{0}\left(Q H^{*}(Y, Z) /\right.$ torsion, $H^{*}(X, Z) /$ torsion) with the set of matrices $A=$ $A_{s_{1}} * A_{s_{2}} * \cdots * A_{s_{1}}$ where

$$
A_{s_{i}}=\left(\begin{array}{c}
A_{s_{i}}^{Q} \\
A_{s_{i}}^{D}
\end{array}\right)
$$

and the order of the matrices $A_{s_{i}}^{Q}, A_{s_{i}}^{D}$ is completely determined by

$$
H^{*}(X, Z) / \text { torsion and } H^{*}(Y, Z) / \text { torsion. }
$$

We denote

$$
A=\left(\begin{array}{l}
A^{Q} \\
A^{D}
\end{array}\right)
$$


3.11. Proposition. Suppose $f: X \rightarrow Y$ is an $H$-map, $H^{*}(X, Q)$ and $H^{*}(Y, Q)$ are primitively generated and $H^{*}(f, Q)$ is either a monomorphism, an epimorphism, an isomorphism or zero.

Given a fibration $F^{\prime \prime} \rightarrow X^{\prime \prime} \stackrel{f^{\prime \prime}}{\rightarrow} Y^{\prime \prime}$ in $G(f)$ and a commutative diagram

$$
\begin{array}{lll}
X^{\prime \prime} & \stackrel{h^{\prime \prime}}{\rightarrow} & X \\
\downarrow f^{\prime \prime} & & \downarrow f \\
Y^{\prime \prime} & \stackrel{k^{\prime \prime}}{\rightarrow} & X
\end{array}
$$

where $h^{\prime \prime}$ and $k^{\prime \prime}$ realize $\tilde{d}=\left( \pm d_{m_{1}}, \ldots, \pm d_{\left.m_{x x}\right)}\right)$ and $\tilde{d}^{\prime}=\left( \pm d_{n_{1}}^{\prime}, \ldots, \pm d_{n_{k y}}^{\prime}\right)$, respectively. Then the map $f^{\prime \prime}: X^{\prime \prime} \rightarrow Y^{\prime \prime}$ is homotopy equivalent to the map $f^{\prime}$ : $X^{\prime} \rightarrow Y^{\prime}$, which corresponds to the pair $\left(d, d^{\prime}\right) \quad\left(d=\left(d_{m_{1}}, \ldots, d_{\left.m_{(x)}\right)}\right), d^{\prime}=\right.$ $\left.\left(d_{n_{1}}^{\prime}, \ldots, d_{n_{(Y)}^{\prime}}^{\prime}\right)\right)$ in the construction of Proposition 3.4.

Remarks. (1) The pair $\left(C_{1}, C_{2}\right)$ which appears in Proposition 3.6 is equal to the matrix

$$
\left(\begin{array}{ll}
C_{1}^{Q} & C_{2}^{Q} \\
C_{1}^{D} & C_{2}^{D}
\end{array}\right)
$$

(2) When we write in the proof functions which correspond to the matrix

$$
\left(\begin{array}{ll}
t_{1} C_{1}^{Q} & t_{2} C_{2}^{Q} \\
t_{3} C_{1}^{D} & t_{4} C_{4}^{D}
\end{array}\right)
$$

where $t\left(f^{\prime}, f^{\prime \prime}\right) / t_{i}$ for every $i$, we mean functions $h: X^{\prime \prime} \rightarrow X^{\prime}, k: Y^{\prime \prime} \rightarrow Y^{\prime}$ so that the matrices of

$$
H^{*}(h, Z) / \text { torsion } \mid\left(P H^{*}\left(X^{\prime}, Z\right) / \text { torsion }\right)
$$

and

$$
H^{*}(k, Z) / \text { torsion } \mid\left(P H^{*}\left(Y^{\prime}, Z\right) / \text { torsion }\right)
$$

are

$$
\left(\begin{array}{ll}
t_{1} & C_{1}^{Q} \\
t_{3} & C_{1}^{D}
\end{array}\right) \text { and }\left(\begin{array}{ll}
t_{2} & C_{2}^{Q} \\
t_{4} & C_{2}^{D}
\end{array}\right)
$$

respectively, and which satisfy $f^{\prime} h \sim k f^{\prime \prime}$. (Such functions exist by Proposition 3.6.)

Proof of Proposition 3.11. We shall prove the proposition in the case $\tilde{d}=d$, $\tilde{d}^{\prime}=d^{\prime}$. The proof in the case $\tilde{d}=\left( \pm d_{m_{1}}, \ldots, \pm d_{m_{k x}}\right) \neq d$ or $\tilde{d}^{\prime}=$ $\left( \pm d_{n_{1}}^{\prime}, \ldots, \pm d_{n_{(Y Y}}^{\prime}\right) \neq d^{\prime}$ is similar.

By Zabrodsky [8] $X^{\prime \prime} \approx X^{\prime}, Y^{\prime \prime} \approx Y^{\prime}$ and $F^{\prime \prime} \approx F^{\prime}$. After localization at $t$ of diagram 3.11.1 and of the outer square of diagram 3.4.1 we obtain a commutative diagram:

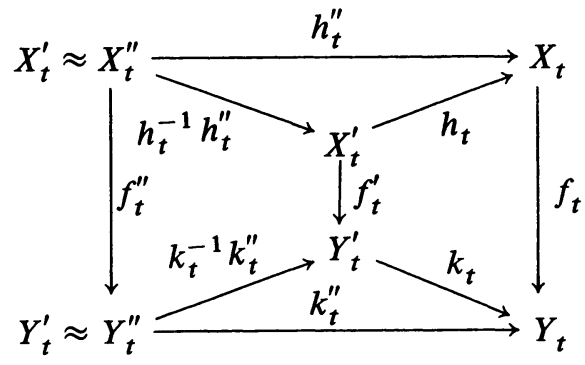


The fact that the left trapezoid is commutative and $f^{\prime}$ is an $H$-map imply (Theorem 2.2) the existence of an integer $n_{1},\left(n_{1}, t\right)=1$, and maps $\tilde{h}: X^{\prime \prime} \rightarrow X^{\prime}, \tilde{k}: Y^{\prime \prime} \rightarrow Y^{\prime}$ so that $f^{\prime} \tilde{h} \sim \tilde{k} f^{\prime \prime}, \tilde{h_{t}} \sim \phi_{n_{1}} h_{t}^{-1} h_{t}^{\prime \prime}$ and $\tilde{k}_{t} \sim \phi_{n_{1}} k_{t}^{-1} k_{t}^{\prime \prime}$. As $h$ and $k$ are $H$-maps $(h \tilde{h})_{t} \sim \phi_{n_{1}} h_{t}^{\prime \prime}$ and $(k \tilde{k})_{t} \sim \phi_{n_{2}} k_{t}^{\prime \prime}$. Therefore there exists an integer $n_{2},\left(n_{2}, t\right)=1$, so that $\phi_{n_{2}}\left(\phi_{n_{1}} h^{\prime \prime}\right) \sim \phi_{n_{2}}(h \tilde{h}) \sim h\left(\phi_{n_{2}} \tilde{h}\right)$ and $\phi_{n_{2}}\left(\phi_{n_{1}} k^{\prime \prime}\right) \sim \phi_{n_{2}}(k \tilde{k}) \sim k\left(\phi_{n_{2}} \tilde{k}\right)$. The maps $h^{\prime}=\phi_{n_{2}} \tilde{h}, k^{\prime}=\phi_{n_{2}} \tilde{k}$ satisfy $\phi_{n} h^{\prime \prime} \sim h h^{\prime}$ and $\phi_{n} k^{\prime \prime} \sim k k^{\prime}\left(n=n_{1} n_{2}\right)$. Consequently the fact that $h$ and $h^{\prime \prime}$ realize $d$ and $k$ and $k^{\prime \prime}$ realize $d^{\prime}$ imply that $h^{\prime}$ and $k^{\prime}$ realize the same as $\phi_{n}$.

Let $\chi: Z H^{*}\left(X^{\prime \prime}, Z\right) /$ torsion $\rightarrow H^{*}\left(X^{\prime \prime}, Z\right)$ be a rational splitting, i.e. the map $Q H^{*}\left(X^{\prime \prime}, Z\right) /$ torsion $\stackrel{x}{\rightarrow} H^{*}\left(X^{\prime \prime}, Z\right) \stackrel{\text { proj }}{\rightarrow} Q H^{*}\left(X^{\prime \prime}, Z\right) /$ torsion is a monomorphism of maximal rank. We shall identify $Q H^{*}\left(X^{\prime \prime}, Z\right) /$ torsion with

$$
\chi\left(Q H^{*}\left(X^{\prime \prime}, Z\right) / \text { torsion }\right) \text {. }
$$

Choose bases for $P H^{*}\left(X^{\prime}, Z\right) /$ torsion and $P H^{*}\left(Y^{\prime}, Z\right) /$ torsion in which $P H^{*}\left(f^{\prime}, Z\right) /$ torsion is represented by a diagonal matrix $M$, and bases for $Q H^{*}\left(X^{\prime \prime}, Z\right) /$ torsion and $Q H^{*}(Y, Z) /$ torsion in which $Q H^{*}\left(f^{\prime \prime}, Z\right) /$ torsion is represented by a diagonal matrix $N^{Q}$. Denote by $N$ the matrix of

$$
H^{*}\left(f^{\prime \prime}, Z\right) / \text { torsion } \mid\left(Q H^{*}\left(Y^{\prime \prime}, Z\right) / \text { torsion }\right)
$$

relative to these bases. Obviously

$$
N=\left(\begin{array}{l}
N^{Q} \\
N^{D}
\end{array}\right)
$$

Let $r$ be the number of generators of $Q H^{*}(X, Q)$ and let $\tilde{r}$ be the number of generators of $Q H^{*}(Y, Q)$. Define $t^{\prime}=\lambda^{2} t\left(f^{\prime}, f^{\prime \prime}\right)$ where $\lambda$ is the multiple of the nonzero elements of $M$ and $N$. Consider the matrices $A^{Q}$ and $\tilde{A}^{Q}$ of $Q H^{*}\left(h^{\prime}, Z\right) /$ torsion and $Q H^{*}\left(k^{\prime}, Z\right) /$ torsion, respectively. Since $h^{\prime}$ and $k^{\prime}$ realize the same as $\phi_{n}$ and $\left(n, t^{\prime}\right)=1$, the matrices $(n I)^{-1} \cdot\left(A^{Q} \otimes Z_{t^{\prime}}\right)$ and $(n I)^{-1}$. $\left(\tilde{A}^{Q} \otimes Z_{t^{\prime}}\right)$ belong to $\operatorname{SL}\left(r, Z_{t^{\prime}}\right)$ and $\operatorname{SL}\left(\tilde{r}, Z_{t^{\prime}}\right)$, respectively. As for every $n$ there exists an epimorphism $\beta_{m}: \operatorname{SL}(m, Z) \rightarrow \operatorname{SL}\left(m, Z_{t^{\prime}}\right)$, there exist matrices $E \in$ $\operatorname{SL}(r, Z), \tilde{E} \in \operatorname{SL}(\tilde{r}, Z)$ so that $\beta_{r}(E)=(n I)^{-1} \cdot\left(A^{Q} \otimes Z_{t^{\prime}}\right)$ and $\beta_{r}(\tilde{E})=(n I)^{-1}$. $\left(A^{Q} \otimes Z_{t^{\prime}}\right.$ ). Consequently there exist matrices $B$ and $\tilde{B}$ (over $Z$ ) so that $A^{Q}=n E$ $+t^{\prime} B$ and $\tilde{A}^{Q}=n \tilde{E}+t^{\prime} \tilde{B}$.

We shall use the conditions on $A^{Q}, \tilde{A}^{Q}$ and $H^{*}(f, Q)$ to construct homotopy equivalences $\bar{h}: X^{\prime \prime} \rightarrow X^{\prime}$ and $\bar{k}: Y^{\prime \prime} \rightarrow Y^{\prime}$ so that $f^{\prime} \bar{h} \sim \bar{k} f^{\prime \prime}$. We shall discuss separately each condition on $H^{*}(f, Q)$.

(a) $H^{*}(f, Q)=0$.

Let $h_{1}: X^{\prime \prime} \rightarrow X^{\prime}, k_{1}: Y^{\prime \prime} \rightarrow Y^{\prime}$ be functions which correspond to the matrix $\left(\begin{array}{cc}-t^{\prime} B & -t^{\prime} B \\ 0 & 0\end{array}\right)$ and let $h_{2}: X^{\prime \prime} \rightarrow X^{\prime}, k_{2}: Y^{\prime \prime} \rightarrow Y^{\prime}$ be functions which correspond to the matrix $\left(\begin{array}{cc}-t^{\prime} B & -t^{\prime} B \\ 0 & 0\end{array}\right)$. Define maps $\bar{h}: X^{\prime \prime} \rightarrow X^{\prime}, \bar{k}: Y^{\prime \prime} \rightarrow Y^{\prime}$ by $\bar{h}=a\left(h^{\prime}+h_{1}\right)+b h_{2}$, $\bar{k}=a\left(k^{\prime}+k_{1}\right)+b k_{2}$, where $a$ and $b$ are integers satisfying $a n+b t^{\prime}=1$. Since the matrices of $Q H^{*}(\bar{h}, Z) /$ torsion and $Q H^{*}(\bar{k}, Z) /$ torsion are $E$ and $\tilde{E}$, respectively, $\bar{h}$ and $\bar{k}$ are homotopy equivalences. Obviously $f^{\prime} \bar{h} \sim \bar{k} f^{\prime \prime}$ ( $f^{\prime}$ is an $H$-map); hence $\bar{h}$ and $\bar{k}$ are the desired maps.

(b) $H^{*}(f, Q)$ is a monomorphism. 
Assume that $A_{s_{i}}^{Q}$ is a $v_{i} \times v_{i}$ matrix and that $\tilde{A}_{s_{i}}^{Q}$ is a $w_{i} \times w_{i}$ matrix. Assume also that for every $i$

$$
M_{s_{i}}=\left(\begin{array}{ccc}
m_{1, s_{i}} & & \\
& \ddots & m_{w_{i}, s_{i}} \\
& & 0
\end{array}\right\}_{v_{i}-w_{i}}
$$$$
\text { and } \quad N_{s_{i}}^{Q}=\left(\begin{array}{ccc}
n_{1, s_{i}} & & \\
& \ddots & \\
& & n_{w_{i}, s_{i}} \\
\hline 0 &
\end{array}\right\}_{v_{i}-w_{i}}
$$

As $A^{Q} M=N^{Q} \tilde{A}^{Q}$ and $\left(\operatorname{det} \tilde{A}_{s_{i}}^{Q}, \lambda\right)=1$, for every $i$ the matrix $A_{s_{i}}^{Q}$ is of the form

$$
A_{s_{i}}^{Q}={ }^{w_{i} \times w_{i}}[\begin{array}{c|c}
C_{s_{i}} & * \\
\hline 0 & \tilde{C}_{s_{i}}
\end{array} \underbrace{}_{\left(v_{i}-w_{i}\right) \times\left(v_{i}-w_{i}\right)}
$$

where $\left|\operatorname{det} C_{s_{i}}\right|=n^{w_{i}}$ and $\left|\operatorname{det} \tilde{C}_{s_{i}}\right|=n^{v_{i}-w_{i}}$. Define

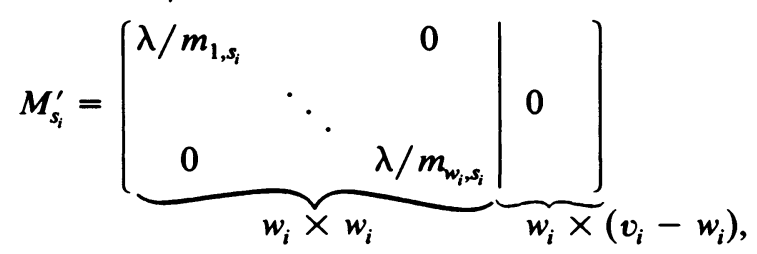

$M^{\prime}=M_{s_{1}}^{\prime} * M_{s_{2}}^{\prime} * \cdots * M_{s_{1}}^{\prime}$ and $\bar{B}=\lambda N \tilde{B} M^{\prime}$. As $\bar{B}$ satisfies

$$
t\left(f^{\prime}, f^{\prime \prime}\right) \bar{B} M=\lambda t\left(f^{\prime}, f^{\prime \prime}\right) N \tilde{B} M^{\prime} M=\lambda t\left(f^{\prime}, f^{\prime \prime}\right) N \tilde{B}(\lambda I)=\lambda^{2} t\left(f^{\prime}, f^{\prime \prime}\right) N \tilde{B}=t^{\prime} N \tilde{B},
$$

there exist maps $h_{1}: X^{\prime \prime} \rightarrow X^{\prime}, k_{1}: Y^{\prime \prime} \rightarrow Y^{\prime}$ which correspond to the matrix

$$
\left(\begin{array}{cc}
-t\left(f^{\prime}, f^{\prime \prime}\right) \bar{B}^{Q} & -t^{\prime} \tilde{B} \\
-t\left(f^{\prime}, f^{\prime \prime}\right) \bar{B}^{D} & 0
\end{array}\right)
$$

Define $h_{2}=h^{\prime}+h_{1}, k_{2}=k^{\prime}+k_{1}$. As $f^{\prime}$ is an $H$-map, $f^{\prime} h_{2} \sim k_{2} f^{\prime \prime}$. From this homotopy and from the definition of $h_{2}$ and $k_{2}$ it follows that the matrices of $Q H^{*}\left(h_{2}, Z\right) /$ torsion and $Q H^{*}\left(k_{2}, Z\right) /$ torsion are $A^{\prime}=A^{Q}-t\left(f^{\prime}, f^{\prime \prime}\right) \bar{B}^{Q}$ and $n \tilde{E}$, respectively, and that $A^{\prime} M=n N^{Q} \tilde{E}$. The last equality together with the facts that $(n, \lambda)=1$ and $\left(\bar{B}_{s_{i}}^{Q}\right)_{k j}=0$ for every $k$ and $j$ that satisfy either $k>w_{i}$ or $j>w_{i}$, imply that for every $i$ the matrix $A_{s_{i}}^{\prime}$ is of the form

$$
A_{s_{i}}^{\prime}={ }^{w_{i} \times w_{i}}\left[\begin{array}{c|c}
n E_{s_{i}}^{\prime \prime} & * \\
\hline 0 & n E_{s_{i}}^{\prime}+t^{\prime} B_{s_{i}}^{\prime}
\end{array}\right]\left(v_{i}-w_{i}\right) \times\left(v_{i}-w_{i}\right)
$$

where $E_{s_{i}}^{\prime} \in \mathrm{GL}\left(v_{i}-w_{i}, Z\right), E_{s_{i}}^{\prime \prime} \in \mathrm{GL}\left(w_{i}, Z\right)$ and $n E^{\prime}+t^{\prime} B_{s_{i}}^{\prime}=\tilde{C}_{s_{i}}$.

For every $i$ denote by $D_{s_{i}}$ the matrix

$$
D_{s_{i}}=w_{i} \times w_{i}\left[\begin{array}{c|c}
0 & t^{\prime} D_{s_{i}^{\prime}}^{\prime} \\
\hline 0 & -t^{\prime} B_{s_{i}^{\prime}}^{\prime}
\end{array}\right]\left(v_{i}-w_{i}\right) \times\left(v_{i}-w_{i}\right)
$$

where $\left(A_{s_{i}}^{\prime}\right)_{k j}+t^{\prime}\left(D_{s_{i}}^{\prime}\right)_{k j} \equiv 0(\bmod n)$ for every $k$ and $j$ that satisfy either $k<w_{i}$ or $j>w_{i}$. Define $D=D_{s_{1}} * D_{s_{2}} * \cdots * D_{s_{i}}$. Since $D M=0$ there exists a function $h_{3}$ : $X^{\prime \prime} \rightarrow X^{\prime}$ so that $f^{\prime} h_{3} \sim *$.

Define functions $h_{4}: X^{\prime \prime} \rightarrow X^{\prime}, k_{4}: Y^{\prime \prime} \rightarrow Y^{\prime}$ by $h_{4}=h_{2}+h_{3}, k_{4}=k_{2}$. It is clear that $f^{\prime} h_{4} \sim k_{4} f^{\prime \prime}$, that the matrix of $Q H^{*}\left(k_{4}, Z\right) /$ torsion is $n \tilde{E}$, that there exists a 
matrix $\bar{E} \in \mathrm{GL}(r, Z)$ so that the matrix of $Q H^{*}\left(h_{4}, Z\right) /$ torsion is $n \bar{E}$ and that $\bar{E} M=N^{Q} \tilde{E}$.

Let $h_{5}: X^{\prime \prime} \rightarrow X^{\prime}, k_{5}: Y^{\prime \prime} \rightarrow Y^{\prime}$ be functions which correspond to the matrix

$$
\left(\begin{array}{cc}
t^{\prime} \bar{E} & t^{\prime} \tilde{E} \\
t\left(f^{\prime}, f^{\prime \prime}\right) \overline{\bar{E}} & 0
\end{array}\right)
$$

where $\overline{\bar{E}}=\lambda N^{D} \tilde{E} M^{\prime}$ (such functions exist since $t^{\prime} N^{Q} \tilde{E}=t^{\prime} \bar{E} M$ and $t^{\prime} N^{D} \tilde{E}=$ $\left.t\left(f^{\prime}, f^{\prime \prime}\right) \overline{\bar{E}} M\right)$. Define $\bar{h}=a h_{4}+b h_{5}, \bar{k}=a k_{4}+b k_{5}$, where $a$ and $b$ are integers satisfying $a n+b t^{\prime}=1$. As the matrices of $\bar{h}$ and $\bar{k}$ are $\bar{E}$ and $\tilde{E}$, respectively, and as $f^{\prime}$ is an $H$-map, $\bar{h}$ and $\bar{k}$ are homotopy equivalences and $f^{\prime} \bar{h} \sim \bar{k} f^{\prime \prime}$.

(c) $H^{*}(f, Q)$ is an epimorphism.

Assume that $A_{s_{i}}^{Q}$ is a $v_{i} \times v_{i}$ matrix, that $\tilde{A}_{s_{i}}^{Q}$ is a $w_{i} \times w_{i}$ matrix and for every $i$

$$
N_{s_{i}}=\underbrace{\begin{array}{ccc|c}
n_{1, s_{i}} & & \\
& \ddots & \\
& & n_{v_{i}, s_{i}}
\end{array}}_{v_{i} \times v_{i}} \underbrace{0}_{v_{i} \times\left(w_{i}-v_{i}\right)}
$$

and

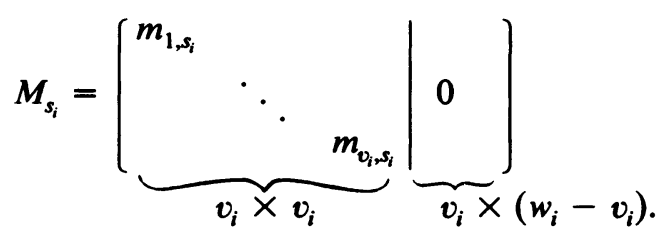

Since $A^{Q} M=N^{Q} \tilde{A}^{Q}$ and $\left(\operatorname{det} A_{s_{i}}^{Q}, \lambda\right)=1$, for every $i$ the matrix $\tilde{A}_{s_{i}}^{Q}$ is of the form

$$
\tilde{A}_{s_{i}}^{Q}={ }^{v_{i} \times v_{i}}\left[\begin{array}{c|c}
C_{s_{i}} & 0 \\
\hline * & \tilde{C}_{s_{i}}
\end{array}\right]\left(w_{i}-v_{i}\right) \times\left(w_{i}-v_{i}\right)
$$

where $\left|\operatorname{det} C_{s_{i}}\right|=n^{v_{i}}$ and $\left|\operatorname{det} \tilde{C}_{s_{i}}\right|=n^{w_{i}-v_{i}}$. Define

$$
N_{s_{i}}^{\prime}=v_{i} \times v_{i}\left(\begin{array}{ccc}
\lambda / n_{1, s_{i}} & & 0 \\
& \ddots & \\
0 & & \lambda / n_{v_{i}, s_{i}} \\
& \left(w_{i}-v_{i}\right) \times v_{i}
\end{array}\right),
$$

$N^{\prime}=N_{s_{1}}^{\prime} * N_{s_{2}}^{\prime} * \cdots * N_{s_{1}}^{\prime}$ and $B^{\prime}=N^{\prime} B M$.

Let $N$ and $\tilde{N}$ be the matrices of $D H^{*}\left(f^{\prime \prime}, Z\right) /$ torsion and $H^{*}\left(f^{\prime \prime}, Z\right) /$ torsion, respectively. As for every decomposable element $d \in H^{*}\left(X^{\prime \prime}, Z\right) /$ torsion there exists a decomposable element $d^{\prime} \in H^{*}\left(Y^{\prime \prime}, Z\right)$ satisfying $\left(f^{\prime \prime}\right) * d^{\prime}=\lambda d$, there exists a matrix $B^{\prime \prime}$ so that $-\lambda N^{D} B^{\prime}=\bar{N} B^{\prime \prime}$. Define $\bar{B}=\left(\begin{array}{c}\lambda B^{\prime} \\ B^{\prime \prime}\end{array}\right), \bar{B}=\left(\begin{array}{l}\bar{B} \\ 0\end{array}\right)$.

As $B^{\prime}$ satisfies

$$
\begin{aligned}
\lambda t\left(f^{\prime}, f^{\prime \prime}\right) N^{Q_{B^{\prime}}} & =\lambda t\left(f^{\prime}, f^{\prime \prime}\right) N^{Q_{N^{\prime}} B M} \\
& =\lambda t\left(f^{\prime}, f^{\prime \prime}\right)(\lambda I) B M=\lambda^{2} t\left(f^{\prime}, f^{\prime \prime}\right) B M=t^{\prime} B M,
\end{aligned}
$$


$\bar{B}$ and $\overline{\bar{B}}$ satisfy $t\left(f^{\prime}, f^{\prime \prime}\right) \tilde{N} \bar{B}=t^{\prime} \overline{\bar{B}} M$. Consequently there exist functions $h_{1}: X^{\prime \prime} \rightarrow$ $X^{\prime}, k_{1}: Y^{\prime \prime} \rightarrow Y^{\prime}$ which correspond to the matrix

$$
\left(\begin{array}{cc}
-t^{\prime} B & -\lambda t\left(f^{\prime}, f^{\prime \prime}\right) B^{\prime} \\
0 & -t\left(f^{\prime}, f^{\prime \prime}\right) B^{\prime \prime}
\end{array}\right)
$$

Define $h_{2}=h^{\prime}+h_{1}, k_{2}=k^{\prime}+k_{1}$. The matrices of $Q H^{*}\left(h_{2}, Z\right) /$ torsion and $Q H^{*}\left(k_{2}, Z\right) /$ torsion are $n E$ and $A^{\prime}=\tilde{A}^{Q}-\lambda t\left(f^{\prime}, f^{\prime \prime}\right) B$. As $f^{\prime} h_{2} \sim k_{2} f^{\prime \prime}, E$ and $A^{\prime}$ satisfy $n E M=N Q_{A^{\prime}}$. This together with the fact that $(n, \lambda)=1$ and $\left(B_{s_{i}}^{\prime}\right)_{k j}=0$ for every pair $(k, j)$ satisfying either $k>v_{i}$ or $j>v_{i}$, imply that for every $i$ the matrix $A_{s_{i}}^{\prime}$ is of the form

$$
A_{s_{i}}^{\prime}=v_{i} \times v_{i}\left[\begin{array}{c|c}
n E_{s_{i}}^{\prime \prime} & 0 \\
\hline 0 & n E_{s_{i}}^{\prime}+t^{\prime} B_{s_{i}}^{\prime}
\end{array}\right]\left(w_{i}-v_{i}\right) \times\left(w_{i}-v_{i}\right)
$$

where $E_{s_{i}}^{\prime} \in \mathrm{GL}\left(w_{i}-v_{i}, Z\right), E_{s_{i}}^{\prime \prime} \in \mathrm{GL}\left(v_{i}, Z\right)$ and $n E_{s_{i}}^{\prime}+t^{\prime} B_{s_{i}}^{\prime}=\tilde{C}_{s_{i}}$.

For every $i$ denote by $D_{s_{i}}$ the matrix

$$
D_{s_{i}}=v_{i} \times v_{i}\left(\begin{array}{c|c}
0 & 0 \\
\hline t^{\prime} D_{s_{i}^{\prime}}^{\prime} & -t^{\prime} B_{s_{i}^{\prime}}^{\prime}
\end{array}\right)\left(w_{i}-v_{i}\right) \times\left(w_{i}-v_{i}\right)
$$

where $\left(A_{s_{i}}^{\prime}\right)_{i j}+t^{\prime}\left(D_{s_{i}}^{\prime}\right)_{i j} \equiv 0(\bmod n)$ for every $i$ and $j$ satisfying either $i>v_{i}$ or $j<v_{i}$. Define $D=D_{s_{1}} * D_{s_{2}} * \cdots * D_{s_{i}}$. Since, for every $i$ and $j, D_{i j}$ is divisible by $\lambda$, there exists a matrix $\bar{D}$ so that $-N^{D} D=\bar{N} \bar{D}$. Denote $D^{\prime}=\left(\frac{D}{\bar{D}}\right)$. $D^{\prime}$ satisfies $\tilde{N} D^{\prime}=0$; therefore there exists a function $k_{3}: Y^{\prime \prime} \rightarrow Y^{\prime}$ so that $k_{3} f^{\prime \prime} \sim$ *

Define $h_{4}: X^{\prime \prime} \rightarrow X^{\prime}$, and $k_{4}: Y^{\prime \prime} \rightarrow Y^{\prime}$ by $h_{4}=h_{2}, k_{4}=k_{2}+k_{3}$. It is obvious that $f^{\prime} h_{4} \sim k_{4} f^{\prime \prime}$, that the matrix of $Q H^{*}\left(h_{4}, Z\right) /$ torsion is $n E$, that there exists a matrix $\bar{E}$ so that the matrix of $Q H^{*}\left(k_{4}, Z\right) /$ torsion is $n \bar{E}$ and that $E M=N^{Q} \bar{E}$.

Let $h_{5}: X^{\prime \prime} \rightarrow X^{\prime}, k_{5}: Y^{\prime \prime} \rightarrow Y^{\prime}$ be functions which correspond to the matrix

$$
\left(\begin{array}{cc}
t^{\prime} E & t^{\prime} \bar{E} \\
0 & t\left(f^{\prime}, f^{\prime \prime}\right) \overline{\bar{E}}
\end{array}\right)
$$

where the matrix $\overline{\bar{E}}$ satisfies $-t^{\prime} N^{D} \bar{E}=t\left(f^{\prime}, f^{\prime \prime}\right) \bar{N} \overline{\bar{E}}$. Define $\bar{h}=a h_{4}+b h_{5}, \bar{k}=$ $a k_{4}+b k_{5}$ where $a$ and $b$ are integers which satisfy $a n+b t^{\prime}=1$. As the matrices of $Q H^{*}(\bar{h}, Z) /$ torsion and $Q H^{*}(\bar{k}, Z) /$ torsion are $E$ and $\bar{E}$, respectively, $\bar{h}$ and $\bar{k}$ are homotopy equivalences. Obviously $f^{\prime} \bar{h} \sim \overline{k f} f^{\prime \prime}$; hence $\bar{h}$ and $\bar{k}$ are the desired maps.

3.12. Corollary. Proposition 3.11 is also true for a map $S^{2 n-1} \rightarrow X$ where $X$ is an $H$-space so that $H^{*}(X, Q)$ is primitively generated.

Proof. The assertion follows from Corollary 3.8 in the same way that Proposition 3.11 follows from Proposition 3.6.

3.13. Corollary. Let $f: X \rightarrow Y$ be a map which satisfies conditions (a) or (b) of Theorem I. Given a map $f^{\prime \prime}: X^{\prime \prime} \rightarrow Y$ in $G_{Y}(f)$ and a t-equivalence $h^{\prime \prime}: X^{\prime \prime} \rightarrow X^{\prime}$ realizing $\tilde{d}=\left( \pm d_{m_{1}}, \ldots, \pm d_{m_{l(X)}}\right)$. Then the map $f^{\prime \prime}: X^{\prime \prime} \rightarrow Y$ is homotopy equivalent to the map $f^{\prime}: X^{\prime} \rightarrow Y$ which corresponds to the pair $(d, 1)\left(d=\left(d_{m_{1}}, \ldots, d_{\left.m_{(X)}\right)}\right)\right.$ by the construction of Proposition 3.4. 
Proof. The corollary is obviously true if $f$ satisfies condition (b), namely if $X=S^{2 n-1}$.

Suppose $f$ satisfies condition (a). By Corollary 2.3, $G_{Y}(f)=0$ if $H^{*}(f, Q)$ is either an isomorphism or an epimorphism. Therefore we have only to check the cases $H^{*}(f, Q)=0$ and $H^{*}(f, Q)$ is a monomorphism.

Choose bases for $P H^{*}\left(X^{\prime}, Z\right) /$ torsion and $P H^{*}\left(Y^{\prime}, Z\right) /$ torsion in which the matrix $A$ of $P H^{*}\left(f^{\prime}, Z\right) /$ torsion is diagonal. Define $t^{\prime}=\lambda t\left(f^{\prime}, f^{\prime \prime}\right)$, where $\lambda$ is the multiple of the nonzero elements of $A$. Assume $\tilde{d}=d$. Using the considerations of Proposition 3.11 one obtains that there exists a map $h^{\prime}: X^{\prime \prime} \rightarrow X^{\prime}$ which realizes the same as $\phi_{n}$ and satisfies $f^{\prime} h^{\prime} \sim \phi_{n} f^{\prime \prime}$, and that the map $f^{\prime \prime}: X^{\prime \prime} \rightarrow Y$ is homotopy equivalent (over $Y$ ) to the map $f^{\prime}: X^{\prime} \rightarrow Y$.

(If $\tilde{d}=\left( \pm d_{m_{1}}, \ldots, \pm d_{m_{(X X)}}\right) \neq d$ the proof is similar.)

3.14. Corollary. Let $f: X \rightarrow Y$ be a map which satisfies the conditions of Theorem III. Given a map $f^{\prime \prime}: X \rightarrow Y^{\prime \prime}$ in $G^{X}(f)$ and a $t$-equivalence $k^{\prime \prime}: Y^{\prime \prime} \rightarrow Y$ realizing $\tilde{d}^{\prime}=\left( \pm d_{n_{1}}, \ldots, \pm d_{\left.n_{(Y)}\right)}\right)$. Then the map $f^{\prime \prime}: X \rightarrow Y^{\prime \prime}$ is homotopy equivalent (under $X)$ to the map $f^{\prime}: X \rightarrow Y^{\prime}$ which corresponds to the pair $\left(1, d^{\prime}\right)\left(d^{\prime}=\left(d_{n_{1}}^{\prime}, \ldots, d_{n_{(X)}}^{\prime}\right)\right)$ by the construction of Proposition 3.4 .

Proof. If $H^{*}(f, Q)$ is either a monomorphism or an isomorphism then $G_{X}(f)=$ 0 by Corollary 2.3. If $H^{*}(f, Q)$ is either an epimorphism or zero, one chooses bases for $Q H^{*}\left(X^{\prime \prime}, Z\right) /$ torsion and $Q H^{*}\left(Y^{\prime \prime}, Z\right) /$ torsion in which the matrix $B$ of $Q H^{*}\left(f^{\prime \prime}, Z\right) /$ torsion is diagonal, then one defines $t^{\prime}=\lambda t\left(f^{\prime}, f^{\prime \prime}\right)$ where $\lambda$ is the multiple of the nonzero elements of $B$. Using the Corollary 3.14 follows from Corollary 3.10 in the same way that Proposition 3.11 follows from Proposition 3.6.

In Theorems I, II and III we referred to an integer $\hat{t}$. We shall define it now:

3.15. Definition. (a) If $f: X \rightarrow Y$ is a map satisfying the conditions of Theorems I or III and $Y \neq S^{2 n-1}$, we define $\hat{t}=t(f, f)$ (of 3.6).

(b) If $X=S^{2 n-1}, Y=S^{2 m-1}(n>m)$ and the order of $f$ is odd we define $\hat{t}=\operatorname{order}(f)$.

(c) If $X=S^{2 n-1}, Y=S^{2 m-1}(n>m)$ and the order of $f$ is even, we define $\hat{t}=|f|^{v}$, where $v$ is an integer satisfying $\eta_{|f|} f \sim *$.

(By the proof of Theorem 2.2 such an integer exists.)

3.16. Proposition. (a) Let $f: X \rightarrow Y$ be a map which satisfies the conditions of Theorem I. There exists a surjection $\xi^{\prime}=\xi_{f}^{\prime}: D \rightarrow G(f)$ satisfying the following conditions:

(1) $\xi^{\prime}\left(d, d^{\prime}\right)=\xi^{\prime}\left(d+\hat{t} s, d^{\prime}+\hat{t s}^{\prime}\right)$ whenever $\left(d, d^{\prime}\right)$ and $\left(d+\hat{t s}, d^{\prime}+\hat{t} s^{\prime}\right)$ belong to $D$.

(2) If $f$ is an $H$-map, then for every pair $\left(d, d^{\prime}\right) \in D, \xi^{\prime}\left(d, d^{\prime}\right)$ is an H-map.

(3) If $D^{\prime}=\{(d, 1) \in D\}$ then $\xi^{\prime} \mid D^{\prime}$ is on $G_{Y}(f)$ and for any two pairs $(d, 1)$, $(d+\hat{t s}, 1)$ in $D^{\prime}, \xi^{\prime}(d, 1)=\xi^{\prime}(d+\hat{t} s, 1)$ in $G_{Y}(f)$.

(b) If the map $f: X \rightarrow Y$ satisfies the conditions of Theorem III and if $D^{\prime \prime}=$ $\left\{\left(1, d^{\prime}\right) \in D\right\}$ then the map $\xi^{\prime} \mid D^{\prime \prime}\left(\xi^{\prime}\right.$ from $\left.(\mathrm{a})\right)$ is on $G^{X}(f)$ and

$$
\xi^{\prime}\left(1, d^{\prime}\right)=\xi^{\prime}\left(1, d^{\prime}+\hat{t} s^{\prime}\right)
$$

in $G_{X}(f)$, whenever $\left(1, d^{\prime}\right)$ and $\left(1, d^{\prime}+\hat{t}^{\prime}\right)$ belong to $D^{\prime \prime}$. 
Proof. Propositions 3.4 and 3.11 imply that there exists a surjection $\xi^{\prime}: D \rightarrow$ $G(f)$, that $\xi^{\prime} \mid D^{\prime}$ and $\xi^{\prime} \mid D^{\prime \prime}$ are on $G_{Y}(f)$ and on $G^{X}(f)$, respectively, and that if $f$ is an $H$-map, then $\xi^{\prime}\left(d, d^{\prime}\right)$ is an $H$-map for every pair $\left(d, d^{\prime}\right) \in D$.

We shall prove part (a)(1) (parts (a)(3) and (b) are proved similarly). We shall distinguish two cases:

(a) $f$ satisfies conditions (a) or (b) of Theorem I.

(b) $f: S^{2 n-1} \rightarrow S^{2 m-1}(n>m)$.

The proof of case (a). It follows from Propositions 3.4 and 3.11 that for every pair $\left(1+\hat{t} s, 1+\hat{t s}^{\prime}\right) \in D, \xi_{f}^{\prime}\left(1+\hat{t} s, 1+\hat{t}^{\prime}\right)=\xi_{f}^{\prime}(1,1)=(f: X \rightarrow Y)$.

Let $\left(d_{1}, d_{1}^{\prime}\right) \in D$ be a pair which satisfies $\left(d_{1} d, d_{1}^{\prime} d^{\prime}\right)=\left(1+\hat{t a}, 1+\hat{t} a^{\prime}\right)$ where $\left(a, a^{\prime}\right) \in Z^{l(Z)} \times Z^{l(Y)}$. Assume that $\xi_{f}^{\prime}\left(d, d^{\prime}\right)=\left(f^{\prime}: X^{\prime} \rightarrow Y^{\prime}\right)$ and that

$$
\xi_{f}^{\prime}\left(d+\hat{t} s, d^{\prime}+\hat{t s^{\prime}}\right)=\left(f^{\prime \prime}: X^{\prime \prime} \rightarrow Y^{\prime \prime}\right) \text {. }
$$

Since $\left(d_{1}(d+\hat{t} s), d_{1}^{\prime}\left(d+\hat{t} s^{\prime}\right)\right)=\left(1+\hat{t}\left(a+d_{1} s\right), 1+\hat{t}\left(a^{\prime}+d_{1}^{\prime} s^{\prime}\right)\right)$, it follows from Proposition 3.4 that $\xi_{f^{\prime}}^{\prime}\left(d_{1}, d_{1}^{\prime}\right)=\xi_{f^{\prime \prime}}^{\prime}\left(d_{1}, d_{1}^{\prime}\right)=f$. Consequently there exists a commutative diagram

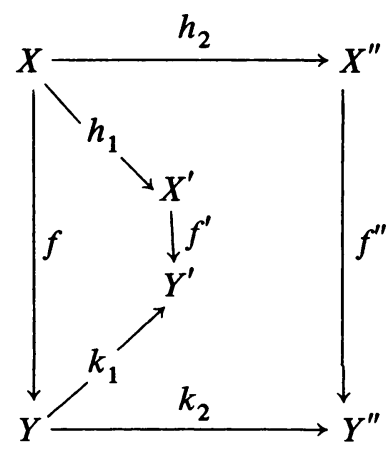

where $h_{1}$ and $h_{2}$ realize $d_{1}$, and $k_{1}$ and $k_{2}$ realize $d_{1}^{\prime}$. Using this diagram we obtain (in the same way that we proved Proposition 3.11) that

$$
\xi^{\prime}\left(d, d^{\prime}\right)=\xi^{\prime}\left(d+\hat{t}, d^{\prime}+\hat{t} s^{\prime}\right)
$$

The proof of (b). Assume that $\xi^{\prime}\left(d, d^{\prime}\right)=\left(f^{\prime}: S^{2 n-1} \rightarrow S^{2 m-1}\right)$ and that $\xi^{\prime}\left(d+\hat{t} s, d^{\prime}+\hat{t} s^{\prime}\right)=\left(f^{\prime \prime}: S^{2 n-1} \rightarrow S^{2 m-1}\right)$. As for every $f^{\prime} \in G(f)$ the order of $f^{\prime}$ is equal to the order of $f$, we obtain from the choice of $\hat{t}$ that $\eta_{d^{\prime}+i s^{\prime}} f^{\prime} \sim f \eta_{d+i s}$; hence $\xi^{\prime}\left(d, d^{\prime}\right)=\xi^{\prime}\left(d+\hat{t} s, d^{\prime}+\hat{t s}^{\prime}\right)$.

3.17. The Proof of Theorems I, II, AND III. We shall prove Theorem I. Theorems II and III are proved similarly.

Let $\beta: D \rightarrow\left[\left(Z_{t}^{*}\right) / \pm 1\right]^{l(X)+l(Y)}$ be the map

$$
\begin{aligned}
\beta\left(d_{m_{1}}, \ldots, d_{\left.m_{k} x\right)}, d_{n_{1}}, \ldots, d_{\left.m_{k \gamma}\right)}\right) \\
\quad=\left(d_{m_{1}}(\bmod \hat{t}), \ldots, d_{\left.m_{k X}\right)}(\bmod \hat{t}), d_{n_{1}}(\bmod \hat{t}), \ldots, d_{n_{k \gamma}}(\bmod \hat{t})\right) .
\end{aligned}
$$


By Proposition 3.16, $\xi^{\prime}: D \rightarrow G(f)$ factors through $\operatorname{Im} \beta$. We shall calculate $\operatorname{Im} \beta$. To this end we shall distinguish among four cases:

(1) $H^{*}(f, Q)=0$. If $\left(d, d^{\prime}\right) \in D$ there is no relation between $d$ and $d^{\prime}$; consequently $\operatorname{Im} \beta=\left[Z_{t}^{*} / \pm 1\right]^{l(X)+l(Y)}$.

(2) $H^{*}(f, Q)$ is an isomorphism. $\left(d, d^{\prime}\right) \in D$ iff $d=d^{\prime}$; consequently

$$
\operatorname{Im} \beta=\left\{(d, d) \in\left[\left(Z_{i}^{*}\right) / \pm 1\right]^{l(X)+l(Y)} \cong\left[Z_{i}^{*} / \pm 1\right]^{l(X)}\right\} .
$$

(3) $H^{*}(f, Q)$ is a monomorphism. Suppose $d=\left(d_{m_{1}}, \ldots, d_{\left.m_{k x}\right)}\right) \in\left[\left(Z_{i}^{*}\right) / \pm 1\right]^{l(X)}$ and $d^{\prime}=\left(d_{n_{1}}^{\prime}, \ldots, d_{n_{(Y)}}^{\prime}\right) \in\left[\left(Z_{i}^{*}\right) / \pm 1\right]^{l(Y)}$. Define $\tilde{d}=\left(\tilde{d}_{m_{1}}, \ldots, \tilde{d}_{m_{(X)}}\right) \in Z^{l(X)}$ by

$$
\tilde{d}_{m_{i}}= \begin{cases}d_{m_{i}}, & m_{i} \neq n_{j} \text { for every } j, \\ c_{i} d_{n_{j}}^{\prime}, & m_{i}=n_{j},\end{cases}
$$

where $0<c_{1}<\hat{t}$ is an integer satisfying $d_{m_{j}} \equiv c_{i} d_{n_{j}}^{\prime}(\bmod \hat{t})$. Obviously $\left(\tilde{d}, d^{\prime}\right) \in D$ and $\beta\left(\tilde{d}, d^{\prime}\right)=\left(d, d^{\prime}\right)$. Consequently $\operatorname{Im} \beta=\left[\left(Z_{i}^{*}\right) / \pm 1\right]^{l(X)+l(n)}$.

(4) $H^{*}(f, Q)$ is an epimorphism. Suppose $d$ and $d^{\prime}$ are as in (3). Define $\tilde{d}=$ $\left(\tilde{d}_{n_{1}}, \ldots, \tilde{d}_{n_{(Y)}}\right) \in Z^{l(Y)}$ by

$$
\tilde{d}_{n_{i}}=\left\{\begin{array}{lc}
d_{n_{i}}^{\prime}, & n_{i} \neq m_{n} \text { for every } j, \\
c_{i} d_{m_{j}}, & n_{i}=m_{j},
\end{array}\right.
$$

where $0<c_{i}<\hat{t}$ is an integer satisfying $d_{n_{j}}^{\prime} \equiv c_{i} d_{m_{j}}(\bmod \hat{t})$. Obviously $(d, \tilde{d}) \in D$ and $\beta(d, \tilde{d})=\left(d, d^{\prime}\right)$. Consequently $\operatorname{Im} \beta=\left[\left(Z_{t}^{*}\right) / \pm 1\right]^{l(X)+l(n)}$.

Define an integer $k$ as follows: If $H^{*}(f, Q)$ is either a monomorphism, an epimorphism or zero put $k=l(X)+l(Y)$ and if $H^{*}(f, Q)$ is an isomorphism put $k=l(X)=l(Y)$. By Proposition 3.16 and the calculation of $\operatorname{Im} \beta$, the surjection $\xi^{\prime}: D \rightarrow G(f)$ induces a surjection $\xi:\left[\left(Z_{i}^{*}\right) / \pm 1\right]^{k} \rightarrow G(f)$. Define an action on $G(f)$ by $\hat{\xi}\left(d, d^{\prime}\right) \cdot \hat{\xi}\left(d_{1}, d_{1}^{\prime}\right)=\hat{\xi}\left(d d_{1}, d^{\prime} d_{1}^{\prime}\right)$. Propositions $3.4,3.11$ and 3.16 imply that the action is well defined, that $G(f)$ with this action is an abelian group and that the sequence

$$
[f, f]_{\hat{t}} \stackrel{\alpha^{\prime}}{\rightarrow}\left[\left(Z_{t}^{*}\right) / \pm 1\right]^{k} \stackrel{\hat{\xi}}{\rightarrow} G(f) \rightarrow 0
$$

is exact.

4. Some consequences of Theorems I, II, and III. We assume that all the maps satisfy the conditions of Theorem I or of Theorem III (Theorem I when we speak of $G(f)$ or $G_{Y}(f)$, Theorem III when we speak of $\left.G^{X}(f)\right)$.

4.1. LemMa. Let $f: S^{2 n-1} \rightarrow S^{2 m-1}(n>m)$ be a map. If the order of $f$ is odd then $G(f)=G_{S^{2 m-1}}(f)=\left[\left(Z_{|f|}^{*}\right) / \pm 1\right]$.

Proof. $\left(d, d^{\prime}\right) \in \operatorname{Im} \alpha^{\prime} \subset\left[\left(Z_{|f|}^{*}\right) / \pm 1\right]^{2}$ if and only if $d \equiv d^{\prime}(\bmod (|f|))$.

REMARK. It is clear that for any map of the form $f: S^{n} \rightarrow S^{n}, G(f)=0$.

4.2. LEMMA. If $f: X \rightarrow Y$ is a map and $f$ is a rational equivalence then each map $f^{\prime}:$ $X^{\prime} \rightarrow Y^{\prime}$ in $G(f)$ is obtained as the pull-back of $X \stackrel{f}{\rightarrow} Y \stackrel{k}{\leftarrow} Y^{\prime}$ where $k$ is a $\hat{t}$-equivalence. In particular for every $f^{\prime} \in G(f), F^{\prime} \approx F$. 
Proof. Follows from the construction that appears in Proposition 3.4.

4.3. LemMa. Let $f_{1}: X_{1} \rightarrow Y_{1}$ and $f_{2}: X_{2} \stackrel{f_{2}}{\rightarrow} Y_{2}$ be maps.

(a) Each map in $G\left(f_{1} \times f_{2}\right)\left(G_{Y_{1} \times Y_{2}}\left(f_{1} \times f_{2}\right), G^{X_{1} \times X_{2}}\left(f_{1} \times f_{2}\right)\right)$ is of the form $g_{1} \times g_{2}$ where $g_{i} \in G\left(f_{i}\right)\left(g_{i} \in G_{Y_{i}}\left(f_{i}\right), g_{i} \in G^{X_{i}}\left(f_{i}\right)\right)$.

(b) If (1) $Q H^{n}\left(X_{1}, Q\right) \neq 0$ whenever $Q H^{n}\left(X_{2}, Q\right) \neq 0$,

(2) $Q H^{n}\left(Y_{1}, Q\right) \neq 0$ whenever $Q H^{n}\left(Y_{2}, Q\right) \neq 0$, then

(1') $G\left(f_{1} \times f_{2}\right)=f_{1} \times G\left(f_{2}\right)$.

(2') $G^{x}\left(f_{1} \times f_{2}\right)=f_{1} \times G^{x}\left(f_{2}\right)$.

(3') $G_{Y}\left(f_{1} \times f_{2}\right)=f_{1} \times G_{Y}\left(f_{2}\right)$.

(4) If $Y_{2}=K\left(Y_{1}\right), X_{2}=K\left(X_{1}\right)$ and $f_{2} \sim *$ then $G\left(f_{1} \times f_{2}\right)=G^{X}\left(f_{1} \times f_{2}\right)=$ $G_{Y}\left(f_{1} \times f_{2}\right)=0$.

4.4. Corollary. Let $f: X \rightarrow Y$ be a map. There exists an integer $n$ so that $G\left(f^{n}: X^{n} \rightarrow Y^{n}\right)=0$.

Proof. If $f^{\prime} \in G(f)$ and $f^{\prime}=\hat{\xi}\left(d, d^{\prime}\right)$ then $\left(f^{\prime}\right)^{n} \in G\left(f^{n}\right)$ satisfies $\left(f^{\prime}\right)^{n}=$ $\hat{\xi}\left(d^{n}, d^{\prime n}\right)$. Consequently $\left(f^{\prime}\right)^{\varphi(t) / 2} \approx f^{\varphi(t) / 2} .(\varphi(\hat{t})=$ the Euler number of $\hat{t}=$ the order of $Z_{l}^{*}$.)

REMARK. It is obvious that the corollary is also true for $G^{X}(f)$ and $G_{Y}(f)$.

4.5. Lemma. Every map in $G($ proj: $X \times Y \rightarrow Y)$ is of the form proj: $X^{\prime} \times Y^{\prime} \rightarrow$ $Y^{\prime}$ where $X^{\prime} \in G(X)$ and $Y^{\prime} \in G(Y)$.

4.6. Lemma. Let $f: X \stackrel{f}{\rightarrow} Y$ be a map $\left(Y \neq S^{2 m-1}\right)$.

(a) Every map in $G\left(\phi_{n} f\right)\left(G^{X}\left(\phi_{n} f\right)\right)$ is of the form $\phi_{n} f^{\prime}$ where $f^{\prime} \in G(f)\left(f^{\prime} \in\right.$ $\left.G^{X}(f)\right)$.

(b) If $X$ is an $H$-space then every map in $G\left(f \phi_{n}\right)\left(G_{Y}\left(f \phi_{n}\right)\right)$ is of the form $f^{\prime} \phi_{n}$ where $f^{\prime} \in G(f)\left(f^{\prime} \in G_{Y}(f)\right)$.

(c) If $X=S^{2 n-1}$ then every map in $G\left(f \eta_{n}\right)\left(G_{Y}\left(f \eta_{n}\right)\right)$ is of the form $f^{\prime} \eta_{n}$ where $f^{\prime} \in G(f)\left(f^{\prime} \in G_{Y}(f)\right)$.

Proof. We shall prove (a). The proofs of (b) and (c) are similar.

Since $f^{*}$ and $\left(\phi_{n} f\right)^{*}$ can be diagonalized simultaneously we can apply diagram 3.4.1 (with the same $\varphi$ and $\psi$ ) to construct $G(f)$ and $G\left(\phi_{n} f\right)$. Suppose $\xi_{f}^{\prime}\left(d, d^{\prime}\right)=\left(X^{\prime} \stackrel{f^{\prime}}{\rightarrow} Y^{\prime}\right)$ and $\xi_{\phi_{n} f}=\left(g^{\prime}: X^{\prime} \rightarrow Y^{\prime}\right)$. From diagram 3.4.1 we obtain that there exist an $H$-map $k: Y^{\prime} \rightarrow Y$ and a map $h: X^{\prime} \rightarrow X$ (obviously if $f$ is an $H$-map $h$ is, also, an $H$-map) which realize $d^{\prime}$ and $d$, respectively, and which satisfy $k g \sim\left(\phi_{n} f\right) h$ and $k f^{\prime} \sim f h$. The last homotopy together with the fact that $k$ is an $H$-map imply that $k\left(\phi_{n} f^{\prime}\right) \sim\left(\phi_{n} f\right) h$. Therefore by Proposition 3.11 the map $\phi_{n} f^{\prime}$ is homotopy equivalent to $g$.

5. The map $G(X, Y, f) \rightarrow G(X) \times G(Y)$. The map $G(X, Y, f) \rightarrow G(X) \times G(Y)$ exists for every map $f: X \rightarrow Y$. An immediate consequence of Theorem $I$ is that for maps $f: X \rightarrow Y$ which satisfy the conditions of Theorem I the above map is a homomorphism and the compositions $G(X, Y, f) \rightarrow G(X) \times G(Y) \stackrel{\text { proj }}{\rightarrow} G(X)$ and $G(X, Y, f) \rightarrow G(X) \times G(Y) \stackrel{\text { proj }}{\rightarrow} G(Y)$ are epimorphisms. 
In this section we deal with the kernel of the map $G(X, Y, f) \rightarrow G(X) \times G(Y)$ only for maps $f: X \rightarrow Y$ which satisfy the conditions of Theorem I. In case that this map is a monomorphism and $G(Y)=0(G(X)=0)$ we conclude (by the previous paragraph) that $G(X, Y, f) \cong G(X)(G(X, Y, f) \cong G(Y))$.

All the notations in the next lemma, except the addition of indices to indicate the dependence in $d$ and $d^{\prime}$, are taken from diagram 3.4.1.

5.1. Lemma. Let $X$ and $Y$ be $H_{0}$-spaces so that $H^{*}(X, Z)$ and $\pi_{*} Y$ are torsion free. If $f: X \rightarrow Y$ is a map which satisfies the conditions of Theorem I then

$$
\begin{aligned}
\mid \operatorname{ker}(G(X, Y, f) & \rightarrow G(X) \times G(Y)) \mid \\
= & \left|\left\{\left[\gamma_{d^{\prime}} g \delta_{d} \psi\right] \in[X, K(Y)] \mid\left(d, d^{\prime}\right) \in D, d_{i}, d_{i}^{\prime}<t^{2}, \forall i\right\}\right|
\end{aligned}
$$

where $\gamma_{d^{\prime}}: K(Y) \rightarrow K(Y)$ and $\delta_{d}: K(X) \rightarrow K(X)$ are homotopy equivalences satisfying $\gamma_{d^{\prime}} \phi_{d^{\prime}} \sim \varphi$ and $\delta_{d} \psi \sim \theta_{d}$.

Proof. It follows from diagram 3.4.1 that if $\xi^{\prime}\left(d, d^{\prime}\right)=f^{\prime}$ then $\phi_{d^{\prime}} f^{\prime} \sim g \in \theta_{d}$. Suppose $\xi^{\prime}\left(d, d^{\prime}\right)$ belongs to $\operatorname{ker}(G(X, Y, f) \rightarrow G(X) \times G(Y))$. The above homotopy together with the homotopies $\gamma_{d^{\prime}} \phi_{d^{\prime}} \sim \varphi$ and $\delta_{d} \psi \sim \theta_{d}$ imply that $\gamma_{d^{\prime}}^{-1} \varphi f^{\prime} \sim$ $g \delta_{d} \psi$ (where $\gamma_{d^{\prime}}^{-1}$ denotes the homotopy inverse of $\gamma_{d^{\prime}}$ ) or equivalently that $\varphi f^{\prime} \sim$ $\gamma_{d^{\prime}} g \delta_{d} \psi$. The truth of the lemma follows from the last homotopy and from the fact that the map $[X, Y] \rightarrow \operatorname{Hom}\left(H^{*}(Y, Q), H^{*}(X, Q)\right)$ is one-to-one (Zabrodsky [9, Lemma 5.3.1]).

REMARK. By 3.17 it is enough to take pairs $\left(d, d^{\prime}\right) \in D$ which satisfy $d_{i}, d_{i}^{\prime}<t^{2}$ for every $i$.

5.2. Examples of maps for which the map $G(X, Y, f) \rightarrow G(X) \times G(Y)$ is a monomorphism. (All the maps considered are assumed to satisfy the conditions of Theorem I.)

EXAMPLE 1. $X \rightarrow K=\prod_{\text {finite }} k\left(Z, n_{i}\right), H^{*}(X, Z)$ is torsion free and $H^{*}(f, Z)$ is onto.

Every $d^{\prime} \in Z^{l(K)}$ can be realized in $K$. Assume that $\left(d, d^{\prime}\right) \in D$ and that $d$ can be realized in $X$. Let $h: X \rightarrow X$ be a map which realizes $d$. As $H^{*}(f, Z)$ is onto $d_{n} / d_{n}^{\prime}$ whenever $Q H^{n}(X, Q) \neq 0$. Consequently there exists a map $k: K \rightarrow K$ which realizes $d^{\prime}$ and satisfies $f h \sim k f$. Therefore by Proposition $3.11, \xi^{\prime}\left(d, d^{\prime}\right)=f$ and the map $G(X, K, f) \rightarrow G(X) \times G(K) \rightarrow G(X)$ is an isomorphism.

EXAMPLE 2. $f: X \rightarrow K(X), f$ is a rational equivalence and $H^{*}(f, Z)$ is onto.

$G(f) \simeq G(X)$, since if $f^{\prime}: X \rightarrow K(X)$ belong to $G(f)$ there exists a pull-back diagram

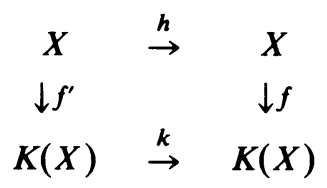

where $h$ and $k$ are $\hat{t}$-equivalences (Lemma 4.2). Consequently $H^{*}\left(f^{\prime}, Z\right)$ is onto and there exists a homotopy equivalence $g: K(X) \rightarrow K(X)$ so that $g f^{\prime} \sim f$.

To state the next two examples one needs the following notations: If $X$ is a CW-complex denote by $h_{n}: X \rightarrow X_{n}$ the homotopy approximation of $X$ in $\operatorname{dim}<n$ (i.e. $\pi_{k} h_{n}$ is an isomorphism for $k \leqslant n$ and $\pi_{k} X_{n}=0$ for $k>n$ ). 
EXAMPLE 3. $h_{n}: X \rightarrow X_{n}$.

Assume that $\left(d, d^{\prime}\right) \in D$ and that $d$ and $d^{\prime}$ can be realized in $X$ and $X_{n}$, respectively. Since if $f: X \rightarrow X$ realizes $d, f_{n}: X_{n} \rightarrow X_{n}$ realizes $d^{\prime}$, one obtains from Proposition 3.11 that $\xi^{\prime}\left(d, d^{\prime}\right)=h_{n}$ and the map $G\left(h_{n}\right) \rightarrow G(X) \times G\left(X_{n}\right)$ is a monomorphism. Moreover, since the map $H^{k}\left(h_{n}, Z\right)$ is an isomorphism in $\operatorname{dim}<$ $n$, the composition $G\left(h_{n}\right) \rightarrow G(X) \times G\left(X_{n}\right) \rightarrow G(X)$ is an isomorphism and $G\left(h_{n}\right)$ $\simeq G(X)$.

EXAMPLE 4. $f: \operatorname{SU}(m) \rightarrow \mathrm{SU}_{2 n-1}, m \leqslant n$, and $H^{*}(f, Z)$ is onto.

Assume that $\left(d, d^{\prime}\right) \in D$, that $d$ and $d^{\prime}$ can be realized in $\mathrm{SU}(m)$ and $\mathrm{SU}_{2 n-1}$, respectively, and that $\xi^{\prime}\left(d, d^{\prime}\right)=g \in G(f)$. Since $f_{\sharp}: \pi_{*} \mathrm{SU}(m) \rightarrow \pi_{*} \mathrm{SU}_{2 n-1}$ is an isomorphism in $\operatorname{dim} \leqslant 2 m-1$ and an epimorphism in $\operatorname{dim} 2 m, g_{\sharp}$ is also an isomorphism in $\operatorname{dim} \leqslant 2 m-1$ and an epimorphism in $\operatorname{dim} 2 m$. Therefore $H^{*}(g, Z)$ is an epimorphism and the fact that the map $G(f) \rightarrow G(\operatorname{SU}(m)) \times$ $G\left(\mathrm{SU}_{2 n-1}\right)$ is a monomorphism follows from the next lemma:

5.3. Lemma. Given maps $f_{1}, f_{2}: \mathrm{SU}(m) \rightarrow \mathrm{SU}_{2 n-1}$ so that $H^{*}\left(f_{1}, Z\right)$ and $H^{*}\left(f_{2}, Z\right)$ are surjections. There exists a homotopy equivalence $g: \mathrm{SU}_{2 n-1} \rightarrow \mathrm{SU}_{2 n-1}$ so that $g f_{1} \sim f_{2}$.

Proof. By Lemma 1.5 in Zabrodsky [7], there exists a map g: $\mathrm{SU}_{2 n-1} \rightarrow \mathrm{SU}_{2 n-1}$ so that $g f_{1} \sim f_{2}$. Obviously $H^{k}(g, Z)$ is an isomorphism for $k<2 m-1$. Assume that $g$ is not a homotopy equivalence and that $k$ is the least integer for which $Q H^{2 k+1}(g, Z) \neq \pm 1$. Consider the diagram

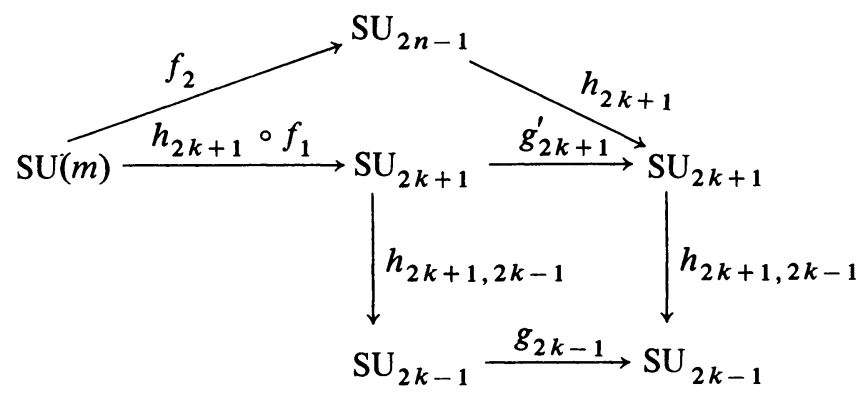

where $g_{2 k+1}^{\prime}$ is a homotopy equivalence which covers the homotopy equivalence $g_{2 k-1}$. (By Zabrodsky [7, Corollary 1.4] such a homotopy equivalence exists.)

As $h_{2 k-1} f_{2} \sim g_{2 k-1} h_{2 k-1} f_{1}$ and the fibration $K(Z, 2 k+1) \rightarrow \mathrm{SU}_{2 k+1} \rightarrow \mathrm{SU}_{2 k-1}$ is principal, there exists $w \in[\mathrm{SU}(m), K(Z, 2 k+1)]$ so that $h_{2 k+1} f_{2} \sim$ $w *\left(g_{2 k+1}^{\prime} h_{2 k+1} f_{1}\right)$ where * is the action of $[\mathrm{SU}(m), K(Z, 2 k+1)]$ on $\left[\mathrm{SU}(m), \mathrm{SU}_{2 n-1}\right]$. Obviously $w$ is decomposable. Since $H^{*}\left(f_{1}, Z\right)$ is onto there exists a decomposable element $\bar{w} \in\left[\mathrm{SU}_{2 k+1}, K(Z, 2 k+1)\right]$ so that $w \sim \bar{w} h_{2 k+1} f_{1}$. Define $g_{2 k+1}^{\prime \prime}=\bar{w} * g_{2 k+1}^{\prime}$. Obviously $g_{2 k+1}^{\prime \prime}$ is a homotopy equivalence and $g_{2 k+1}^{\prime \prime} h_{2 k+1} f_{1} \sim h_{2 k+1} f_{2}$. Consequently $g_{2 k+1}^{\prime \prime}$ can be lifted to a homotopy equivalence $g^{\prime \prime}: \mathrm{SU}_{2 n-1} \rightarrow \mathrm{SU}_{2 n-1}$ so that $g^{\prime \prime} f_{1} \sim f_{2}$. 
6. Computation of $G(a), G_{Y}(a)$ and $G_{K(G, n)}(a)$ for some fibrations $X \stackrel{f}{\rightarrow} Y \stackrel{a}{\rightarrow} K(G, n)$. We assume that all the fibrations in this section are Hopf fibrations which satisfy the conditions of Theorem I.

In order to calculate $G(a), G_{Y}(a)$ and $G_{K(G, n)}(a)$ (in some of the cases) we need the following lemma:

6.1. LEMMA. If the fibration $K(G, n-1) \rightarrow X \stackrel{f}{\rightarrow} Y$ is innduced by $a: Y \rightarrow K(G, n)$ then $G(f) \cong G(a)$ and $G_{Y}(f) \cong G_{Y}(a)$.

Proof. Suppose $X^{\prime} \stackrel{f^{\prime}}{\rightarrow} Y^{\prime} \stackrel{a^{\prime}}{\rightarrow} K(G, n)$ belongs to $G(a)$. Define maps $h: G(a) \rightarrow$ $G(f)$ by $a^{\prime} \rightarrow f^{\prime}$ where $f^{\prime}$ is the fiber of $a^{\prime}$ and $k: G(f) \rightarrow G(a)$ by $f^{\prime} \rightarrow a^{\prime}$ where $f^{\prime}$ is induced by $a^{\prime}$. As each of the maps $h$ and $k$ is the inverse of the other $G(f) \simeq G(a)$. The fact that $G_{Y}(a) \cong G_{Y}(f)$ is proved similarly.

Case $1 . F \rightarrow K(Z, m) \stackrel{a}{\rightarrow} K(Z, n)$.

Obviously $G(a)=0$ for $n<m$. Assume that $n>m$ and that $\left(d, d^{\prime}\right) \in D \subseteq Z^{2}$. Since $H^{n}(a, Q)=0$ there is no relation between $d$ and $d^{\prime}$. In contrast with this, the existence of maps $h: K(Z, m) \rightarrow K(Z, m)$ and $k: K(Z, n) \rightarrow K(Z, n)$ satisfying $a h \sim k a$ implies that $d \equiv d^{\prime}(\bmod (|a|))$. Consequently $G(a) \simeq\left[\left(Z_{|a|}^{*}\right) / \pm 1\right]$.

For the same reason $G_{K(Z, n)}(a) \cong G_{K(Z, m)}(a) \cong\left[\left(Z_{|a|}^{*}\right) / \pm 1\right]$.

Case 2. $F \rightarrow K\left(Z^{m}, n\right) \stackrel{a}{\rightarrow} K\left(Z^{l}, n\right), k, m \geqslant 1$.

$G(a)=0$, since one can choose bases for $H^{n}\left(K\left(Z^{l}, n\right), Z\right)=Z^{l}$ and $H^{n}\left(K\left(Z^{m}, n\right) Z\right)=Z^{m}$ in which $H^{n}(a, Z)$ is represented by a diagonal matrix and use these bases together with the conditions on $D$ to construct for every pair $\left(d, d^{\prime}\right) \in D$ maps $h: K\left(Z^{m}, n\right) \rightarrow K\left(Z^{m}, n\right)$ and $k^{\prime}: K\left(Z^{\prime}, n\right) \rightarrow K\left(Z^{\prime}, n\right)$ which realize $d$ and $d^{\prime}$, respectively and satisfy $a h \sim k a$.

In the same way we obtain that $G_{K\left(Z^{\prime}, n\right)}(a)=G_{K\left(Z^{m}, n\right)}(a)=0$.

Case 3. $F \rightarrow K\left(Z^{l}, n\right) \rightarrow K\left(Z_{p^{k}}, n\right)(k, l>1)$.

We prove that $G(a)=0$ by constructing to each vector $\left(x_{1}, \ldots, x_{l}\right)\left(x_{i} \in Z_{p^{k}}\right)$ and to each number $d \in Z_{p^{k}}$ an $l \times l$ matrix $A$ (over $Z$ ) so that $\operatorname{det} A=d$ and $A\left(x_{1}, \ldots, x_{l}\right) \equiv\left(d x_{1}, \ldots, d x_{l}\right)\left(\bmod p^{k}\right)$.

Consider the vector $\left(x_{1}, \ldots, x_{l}\right)$. Each $x_{i}$ is of the form $x_{i}=a_{i} p^{k_{i}}$ where $(a, p)=1$. Without loss of generality assume that $k_{1}<k_{i}$ or every $i$. Let $b$ be an integer satisfying $a_{1} b \equiv 1\left(\bmod p^{k}\right)$ and let $A=\left(a_{i j}\right)$ be the following matrix:

$$
A=\left(\begin{array}{ccccc}
d & & & \\
\vdots & & 0 & \\
b(d-1) a_{i} p^{k_{i}-k_{1}} & 1 & & \\
& & \ddots & \\
\vdots & 0 & & 1
\end{array}\right)
$$


namely

$$
a_{i j}= \begin{cases}d, & i=1, j=1, \\ b(d-1) a_{i} p^{k_{i}-k_{1},}, & i \neq 1, j=1, \\ 1, & i \neq 1, j=i, \\ 0, & \text { otherwise. }\end{cases}
$$

Obviously $\operatorname{det} A=d$ and $A\left(x_{1}, \ldots, x_{l}\right) \equiv\left(d x_{1}, \ldots, d x_{l}\right)\left(\bmod p^{k}\right) .\left(a_{i 1} x_{1} \equiv\right.$ $(d-1) x_{i}\left(\bmod p^{k}\right)$ for $i>1$.)

Case 4. $F \rightarrow K(G, m) \rightarrow K(H, m), G$ and $H$ are finite $p$-groups.

It is obvious that $G(a) \cong G^{K(G, m)}(a) \cong G_{K(H, m)}(a)=0$.

Case 5. $X_{n+1} \stackrel{h_{n}}{\rightarrow} X_{n} \stackrel{a}{\rightarrow} K(G, n+2)$, the Postnikov approximation of $X$.

It follows from Lemma 6.1 and from Example 3 in $\$ 5$ that $G(a) \cong G\left(h_{n}\right) \cong$ $G\left(X_{n+1}\right)$.

Case 6. $X \stackrel{f}{\rightarrow} Y \stackrel{a}{\rightarrow} K(G, n), \pi_{n} a$ is an epimorphism.

Suppose $X \stackrel{f^{\prime}}{\rightarrow} Y \stackrel{a}{\rightarrow} K(G, n)$ is in $G_{Y}(a)$. Let $k, k^{\prime}: X \rightarrow X$ be $\hat{t}$-equivalences satisfying $f k \sim f^{\prime}$ and $f^{\prime} k^{\prime} \sim f$ (by Theorem 2.2 such $\hat{t}$-equivalences exist). We shall prove that $G_{Y}(a) \cong G(X)$ by showing that $k$ is a homotopy equivalence.

It is obvious that $k_{\sharp}: \pi_{m} X \rightarrow \pi_{m} X$ is an isomorphism for $m \neq n, n-1$ and that $\pi_{n} k$ and $\pi_{n} f$ are monomorphisms. As $\pi_{n} a$ is onto $\pi_{n-1} k$ is also an isomorphism. Consequently in order to prove that $k$ is a homotopy equivalence it is enough to prove that $\pi_{n} k$ is an epimorphism. But $\pi_{n} f \circ \pi_{n} k=\pi_{n} f^{\prime}$ and $\pi_{n} f^{\prime} \circ \pi_{n} k^{\prime}=\pi_{n} f$; hence $\pi_{n} f\left(\pi_{n} k \circ \pi_{n} k^{\prime}\right)=\pi_{n} f, \pi_{n} k \circ \pi_{n} k^{\prime}=1$ and $\pi_{n} k$ is an epimorphism.

Case 7. $X \rightarrow Y \stackrel{a}{\rightarrow} K(G, n), G$ is a finitely generated free group, $H^{n}(Y, Z)$ is torsion free and $H^{n}(a, Z)$ is a surjection.

Assume that $d=\left(d_{1}, \ldots, d_{l(Y)}\right) \in Z^{l(Y)}$ can be realized by a map $h: Y \rightarrow Y$ and that $\left(d, d^{\prime}\right) \in D \subseteq Z^{l(Y)+1}$. As ker $H^{n}(a, Z)=0$ implies $d_{n}=d^{\prime}$ and ker $H^{n}(a, Z) \neq 0$ implies $d_{n} / d^{\prime}$, there exists a map $k: K(G, n) \rightarrow K(G, n)$ which realizes $d^{\prime}$ and satisfies $k a \sim a h$. Hence by Proposition $3.11, \xi^{\prime}\left(d, d^{\prime}\right)=a$ and consequently $G(a) \cong G(Y)$.

7. Noncancellation. In the following proposition we use the notations of Theorem 3.2 .

7.1. Proposition. Let $X$ and $Y$ be $H_{0}$-spaces so that $Q H^{n_{1}}(X, Q) \neq 0$ for $1<i<l(X)$ and $Q H^{m}(Y, Q) \neq 0$ for $1<i<l(Y)$, and let $\psi: X \rightarrow K(X)$ and $\varphi$ : $Y \rightarrow K(Y)$ be rational equivalences. Denote by $t$ the least common multiple of $t(X, \psi)$ and $t(Y, \varphi)$. Assume that

$$
\begin{aligned}
d & =\left(d_{n_{1}}, \ldots, d_{n_{X X)}}\right) \in\left[\left(Z_{t}^{*}\right) / \pm 1\right]^{l(X)} \text { and } \\
d^{\prime} & =\left(d_{m_{1}}^{\prime}, \ldots, d_{\left.m_{X Y}\right)}^{\prime}\right) \in\left[\left(Z_{t}^{*}\right) / \pm 1\right]^{l(Y)}
\end{aligned}
$$


satisfy the following conditions:

(a) $\quad d_{m_{i}}^{\prime}= \begin{cases}d_{n_{j}}, & m_{i}=n_{j}, \\ 1, & m_{i} \neq n_{j} \text { for every } j .\end{cases}$

(b) $d_{n_{i}}= \begin{cases}d_{m}^{\prime}, & n_{i}=m_{j}, \\ 1, & n_{i} \neq m_{j} \text { for every } j .\end{cases}$

If $X^{\prime}=\xi(d) \in G(X)$ and $Y^{\prime}=\xi\left(d^{\prime}\right) \in G(Y)$ then $X^{\prime} \times Y \approx X \times Y^{\prime}$.

Proof. It follows from the definition of $\xi$ that if $Y^{\prime}=\xi\left(d^{\prime}\right) \in G(Y)$ then $Y=\xi\left(\left(d^{\prime}\right)^{-1}\right) \in G\left(Y^{\prime}\right)$. Consequently $X^{\prime} \times Y=\xi(1, \ldots, 1) \in G\left(X \times Y^{\prime}\right)$ and $X^{\prime} \times Y \approx X \times Y^{\prime}$

An immediate consequence of this proposition is

7.2. Corollary. Let $X$ be an $H$-space and let $Y$ be an $H_{0}$-space. If $l(X)=l(Y)$ +1 then for every $X^{\prime} \in G(X)$ there exists a $Y^{\prime} \in G(Y)$ so that $X^{\prime} \times Y \approx X \times Y^{\prime}$.

Using Corollary 7.2 together with Theorem 2.2 one obtains

7.3. Lemma. Let $F \stackrel{j}{\rightarrow} X \stackrel{f}{\rightarrow} Y$ be a fibration satisfying the conditions of Theorem $I$.

(a) For every fibration $F^{\prime} \stackrel{j^{\prime}}{\rightarrow} X^{\prime} \stackrel{f^{\prime}}{\rightarrow} Y$ in $G_{Y}(f), X \times F^{\prime} \approx X^{\prime} \times F$.

(b) If $f$ is a rational equivalence then for every fibration $F^{\prime} \stackrel{j^{\prime}}{\rightarrow} X^{\prime} \stackrel{f^{\prime}}{\rightarrow} Y^{\prime}$ in $G(f)$, $X \times Y^{\prime} \approx X^{\prime} \times Y$.

Proof. (a) Choose bases for $\pi_{*} X /$ torsion and $\pi_{*} Y /$ torsion in which $\pi_{*} f /$ torsion is represented by a diagonal matrix $A$. Let $t$ be an integer divisible by $\left|{ }^{t} \pi_{n} X\right| \cdot\left|{ }^{t} \pi_{n} Y\right|$ for $n<\max \{N(X), N(Y)\}$ and by the nonzero elements of $A$. By Theorem 2.2 there exist $t$-equivalences $h: X^{\prime} \rightarrow X$ and $k: F^{\prime} \rightarrow F$ so that $f h \sim f^{\prime}$ and $j k \sim h j^{\prime}$.

The choice of $t$ together with the commutativity of the diagram

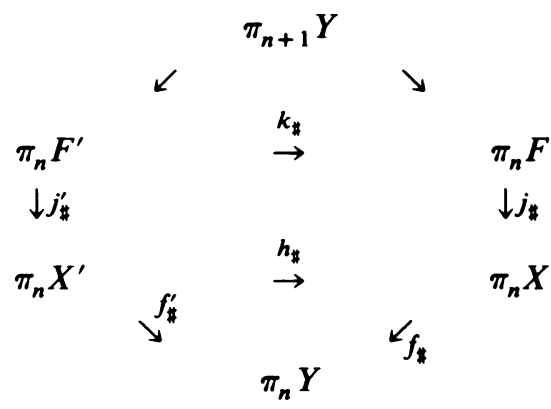

imply that $\operatorname{det}\left(k_{\sharp} \mid \operatorname{ker} j_{\sharp}^{\prime}\right)=1, \operatorname{det}\left(h_{\sharp} \mid\left[\left(\pi_{*} X^{\prime} /\right.\right.\right.$ torsion $\left.\left.) / \operatorname{ker} f_{\sharp}^{\prime}\right]\right)=1$ and

$$
\left.\operatorname{det}\left(h_{\sharp} \mid \operatorname{ker} f_{\sharp}^{\prime}\right)=\operatorname{det}\left(k_{\sharp} \mid\left(\pi_{*} F^{\prime} / \text { torsion }\right) / \operatorname{ker} j_{\sharp}\right]\right) \text {. }
$$

Consequently one obtains from Proposition 7.1 that $X \times F^{\prime} \approx X^{\prime} \times F$.

(b) Let $t$ be as in 3.1 and let $h: X^{\prime} \rightarrow X, k: Y^{\prime} \rightarrow Y$ be $t$-equivalences satisfying $f h \sim k f^{\prime}$ (by Theorem 2.2 such $t$-equivalences exist). Since $\operatorname{det}\left(Q H^{*}(h, Z) /\right.$ torsion) $=\operatorname{det}\left(Q H^{*}(k, Z) /\right.$ torsion $), X^{\prime} \times Y \approx X \times Y^{\prime}$. 


\section{REFERENCES}

1. M. Arkowitz, Localization and $H$-spaces, Lecture Notes Series, No. 44, Matematisk Institut, Aarhus Univ., Aarhus, 1976.

2. C. R. Curjel, On H-space structure of finite complexes, Comment. Math. Helv. 43 (1967), 1-17.

3. P. Hilton, G. Mislin and J. Roitberg, Localization in nilpotent groups and spaces, North-Holland Math. Studies, vol. 15, North-Holland, Amsterdam, 1975.

4. M. Mimura and H. Toda, On p-equivalences and p-universal spaces, Comment. Math. Helv. 46 (1971), 87-97.

5. G. Mislin, The genus of an $\boldsymbol{H}$-space, Symposium on Algebraic Topology, Lecture Notes in Math., vol. 249, Springer-Verlag, Berlin and New York, 1971, pp. 75-83.

6. Cancellation properties of H-spaces, Comment. Math. Helv. 49 (1974), 195-200.

7. A. Zabrodsky, On the homotopy type of principal classical group bundles over spheres, Israel J. Math. 11 (1972), 315-325.

8. Lecture Notes in Math., vol. 418, Springer-Verlag, Berlin and New York, 1974, pp. 161-171.

9. __ Hopf spaces, North-Holland Math. Studies, vol. 22, North-Holland, Amsterdam, 1976.

Department of Mathematics, Bar-Ilan University, RAMAt-Gan, ISRAEl 